\title{
Reducing inefficiency in public good provision through linking
}

\author{
Stefano Galavotti* \\ Dipartimento di Matematica per le Decisioni \\ Università degli Studi di Firenze, Italia
}

February 2010

\begin{abstract}
We derive the optimal mechanism for the provision of $n$ identical public goods in an economy with two agents, binary valuations. The mechanism "links" the $n$ problems together because decisions and transfers are based on the whole vector of valuations of the agents. In particular, if agents have mixed valuations for a public good (one agent has a high valuation, the other a low valuation), the good is provided if the low valuation agent has a sufficiently high average valuation for the whole bundle of goods. We show that the mechanism is asymptotically efficient and we provide an example which compares the gains in terms of efficiency with optimal separate provision and with another asymptotically efficient mechanism in the literature.
\end{abstract}

Keywords: public goods, linking, bundling.

JEL Classification: D61, H21, H41.

${ }^{*}$ E-mail address: stefano.galavotti@unifi.it, sgalavotti@unive.it. 


\section{Introduction}

Inefficiency in public good provision is a well known problem in economics. In general, when the decision is decentralized, free riding behavior leads to an underprovision of public goods. Besides, even if the decision is centralized, the presence of asymmetric information might make impossible to provide the efficient level of public goods. This occurs when the central agency, besides achieving efficiency, needs the good to be self-financing and has no coercive power or does not want to exert it (see Mailath and Postlewaite (1990)) ${ }^{1}$.

This problem is not peculiar to public good provision. When participation and budget balance constraints are imposed, very often efficiency and incentives become impossible to reconcile.

One solution suggested in the literature to alleviate this conflict is that of linking or bundling several decisions together. The idea is the following: suppose there are $n$ different social choice problems and that the objective is to take a socially optimal decision on them; instead of treating each problem separately, we might link them, making the decision on each single problem dependent on all the others. In other words, the $n$ problems can be viewed as a single bundle and incentives can be pooled to obtain truthtelling at a lower cost in terms of inefficiency ${ }^{2}$.

This idea is applied by Armstrong (1999) to the problem of finding an (almost) optimal pricing scheme for a multi-product monopolist ${ }^{3}$ and by Armstrong (2000) to the problem of designing a revenue maximizing multi-object auction. In the former, linking takes the form of a two-part tariff, in which consumers have to pay a fixed charge to be able to buy any product (at marginal cost); in the latter, linking takes the form of a particular tie breaking rule: when bidders make the same (low) bid for any object, then the object is assigned to the bidder who bid highest for the other object.

In the context of public good provision, which is the one we consider here, a few papers have studied the possibility of linking. Quite surprisingly, most of these papers consider excludable public goods, i.e. goods whose usage by consumers can be somehow excluded. These papers include Hellwig $(2004,2007)$ and Fang and Norman (2008). The possibility of excluding consumers from the benefits of the public goods makes this problem similar to the problem of a multi-product monopolist who produces a number of private goods (the accesses to each public good) which are characterized by a fixed cost of production but no variable costs. It is then not surprising that the optimal solution involves exclusion of low valuation consumers (see Fang and Norman (2008)), just like the optimal pricing scheme of a multi-product monopolist has some consumers

\footnotetext{
${ }^{1}$ Hellwig (2007) points out that, even if the central agency has the power to impose her decision and the relative financing scheme on the agents, she might not want to exert it for equity reasons: in fact, the use of coercion to obtain contributions from people who do not care of the public good makes an implicit redistribution of welfare from these people to those who really benefit from it.

${ }^{2}$ The term bundling has its roots in the industrial organization literature: it refers to nonlinear pricing schemes in which several goods or several units of the same good can only be purchased together (in bundles). In more general contexts, the term linking seems more appropriate, but it rests on the same idea

${ }^{3}$ Early contributions on commodity bundling by a monopolist include Adams and Yellen (1976), Palfrey (1983), McAfee et al. (1989) and Armstrong (1996). More recent papers include Bakos and Brynjolfsson (1999), Geng, Stinchcombe and Whinston (2005) and Fang and Norman (2006b).
} 
with low willingness to pay not buying any goods (see Armstrong (1996)).

When exclusion from usage is feasible, the central agency has an additional instrument at her disposal to alleviate the free riding problem: in fact, the threat of individual exclusion makes participants more willing to contribute to the financing of the public good. Therefore, when first best outcomes cannot be implemented, the possibility of exclusion makes it easier to achieve good second best outcomes. In many cases, however, such an exclusion is technologically impossible or, at least, extremely costly: think, for example, to the provision of national defense or to projects that improve air quality or the beauty of a town. It is then interesting to investigate how optimal linking looks like in the context of non-excludable public goods.

There are also two recent papers that show how linking may help approaching first best outcomes in a general mechanism design setting. Jackson and Sonnenschein (2007) consider $n$ identical copies of a general social choice problem and construct a mechanism that is asymptotically efficient. In their mechanism, the message space is rationed in the sense that agents must report a vector of messages that matches the theoretical frequency distribution. In some sense, this relaxes the incentive problems simply by deleting some incentive compatibility constraints. Fang and Norman (2006a), instead, show that a standard Groves mechanism amended with a veto game produces an outcome which is asymptotically efficient. However, this mechanism might perform poorly when the number of problems is small; moreover, it is budget balanced only for a sufficiently large number of problems.

In this paper, we apply the idea of linking to the problem of providing multiple public goods in a two-agent economy. Unlike most of the related contributions, we concentrate on the standard notion of public goods, namely goods that are non-rival and non-excludable. We restrict our attention to the situation in which first best (i.e. efficient) outcomes are not achievable and derive the optimal linking mechanism for the provision of $n$ public goods. This mechanism "links" the $n$ problems together because decisions and transfers are based on the whole vector of valuations of the agents. In particular, if agents have mixed valuations for a public good (one agent has a high valuation, the other a low valuation), the good is provided if the low valuation agent has a sufficiently high average valuation for the whole bundle of goods. This generates an increase in total expected welfare with respect to the optimal single-good mechanism, by making it unlikely to take an inefficient decision. The role of transfers is crucial in driving the result: they transfer surplus from agents with a low average valuation for the public goods to those with a high average valuation, thus giving to the latter the right incentives to be truthful. We show that the mechanism is asymptotically efficient in the sense that the probability that an inefficient decision is taken vanishes as the number of linked problems increases. Finally, we compare by means of an example the performance of our mechanism with the asymptotically efficient mechanism by Fang and Norman (2006a). We show that the performance of this mechanism might be relatively poor when the number of problems is small. Instead, our mechanism is optimal in the sense that maximizes expected social welfare for all $n$. Thus, when the number of available problems is limited, our mechanism might yields a substantial increase in welfare with respect to the asymptotically efficient mechanism by Fang and Norman (2006a).

The rest of the paper is organized as follows: Section 2 introduces the model; 
Section 3 formalizes our problem, showing also that linking cannot restore full efficiency; Section 4 presents our main result, which is the optimal (2nd best) linking mechanism; in Section 5, the properties of such a mechanism are described and an illustrating example is provided. Section 6 briefly concludes.

\section{The model}

Consider the following collective choice problem: A society is made of two agents (labeled \#1 and \#2) and has to decide whether or not to produce $n$ identical public goods (or projects). Denote by $\mathcal{N}=\{1,2, \ldots, n\}$ the set of such goods. The cost of each public good is constant and denoted by $c>0$. Agent $i$ attaches a value $v_{i}^{k}$ to good $k$ being provided, where $\mathbf{v}_{\mathbf{i}}=\left(v_{i}^{k}\right)_{k=1}^{n}$ is private information. We assume that $v_{i}^{k},(i=1,2 ; k \in \mathcal{N})$ are i.i.d. random variables with support $V=\{\underline{v}, \bar{v}\}$, where $\underline{v}<\bar{v}$ and that $\alpha \in(0,1)$ is the probability that $v_{i}^{k}=\underline{v}$. We thus assume independence not only across agents, but also across public goods. We often refer to $\underline{v}$ and $\bar{v}$ simply as low and high valuation respectively. Preferences are linear in the goods and money, i.e. $u_{i}=\sum_{k=1}^{n} p^{k} v_{i}^{k}+t_{i}$, where $t_{i}$ is any money transfer to agent $i, p^{k}=1$ if the $k$-th good is produced and $p^{k}=0$ if it is not ${ }^{4}$. In the sequel, we will also allow for uncertain decisions (i.e. $p^{k} \in[0,1]$ ); utilities have thus to be intended as Von Neumann-Morgestern expected utilities. All of the above is common knowledge.

The linking mechanism we look for consists of two parts: whether or not to provide each of the $n$ public goods (more generally, the probability of providing them); and a vector of (positive or negative) monetary payments to the agents. Invoking the revelation principle, we will restrict our attention to Bayesian incentive compatible (IC) direct revelation mechanisms, i.e. mechanisms in which agents, in a Bayesian equilibrium, truthfully report their private information. The decisions and payments are thus made contingent on such reports by the agents. Formally, the linking mechanism consists of:

(i) a decision function $\mathbf{p}: V^{n} \times V^{n} \rightarrow[0,1]^{n}$, that associates to each pair of vectors of valuations reported by the two agents, a vector of probabilities $\mathbf{p}=\left(p^{k}\right)_{k=1}^{n}$, where $p^{k}$ is the likelihood of producing good $k ;$

(ii) a transfer rule $\mathbf{t}: V^{n} \times V^{n} \rightarrow \mathbb{R}^{2}$, that determines two monetary payments, one to agent $1\left(t_{1}\right)$ and one to agent $2\left(t_{2}\right)$.

A reasonable property that a public good provision mechanism ought to satisfy is that the cost of the goods that are produced has to be recovered through the contributions of the agents. We formalize this property by introducing an ex ante budget balance constraint (BB). Ex ante budget balance only requires that expected payments cover expected costs; however, when types are independent and participation decisions are made at the interim stage, as in our case, we can always find another payment scheme that satisfy the stronger requirement of ex post budget balance (see Börgers and Norman (2006)).

Clearly, an agent is willing to contribute to the provision of a public good only if the benefit he gets from such good exceeds the contribution he is required to pay. Hence, when the central agency has no coercive power, she has to make

\footnotetext{
${ }^{4}$ Notice that there are no complementarities among different public goods.
} 
sure that all agents voluntarily agree on the financing scheme proposed. Besides, even though the central agency has the power to impose her decision (e.g. the government), she might not want to exert it: by doing so, even people who do not benefit from the public goods at all might be required to finance it. This would raise equity concerns. We thus require that the mechanism is such that each agent's expected net benefit is nonnegative, i.e. we impose an interim individual rationality constraint (IR).

We will say that a mechanism is incentive feasible if it simultaneously satisfies $\mathrm{IC}, \mathrm{BB}$ and IR.

\subsection{Anonymity and Label Free}

We are going to restrict our attention to anonymous and label free mechanisms, as defined below. These restrictions, besides simplifying matters, respond to reasonable equity and symmetry considerations. Moreover, as it is shown in Fang and Norman (2008), they do not involve any loss of generality, in the sense that, for any incentive feasible mechanism, there exists another incentive feasible, anonymous and label free mechanism that yields the same social surplus.

a) Anonymity (or Equal Treatment). The decision about providing any public good depends only on the reported valuations but not on "who reports what"; similarly, the payment to agent 1 when she reports $\mathbf{x}$ and agent 2 reports $\mathbf{y}$ is the same as the payment to agent 2 when she reports $\mathbf{x}$ and agent 1 reports y. Formally:

$$
\mathbf{p}(\mathbf{x} ; \mathbf{y})=\mathbf{p}(\mathbf{y} ; \mathbf{x}), \quad t_{1}(\mathbf{x} ; \mathbf{y})=t_{2}(\mathbf{y} ; \mathbf{x}), \quad \forall \mathbf{x}, \mathbf{y} \in V^{n}
$$

b) Label Free: the label attached to each good is irrelevant in the sense that the mechanism gives rise to the same outcome after we reshuffle the labels of the goods. Formally, let $\mathcal{N}=\{1,2, \ldots, n\}$ be the set of public goods and $\pi: \mathcal{N} \mapsto \mathcal{N}$ a permutation of this $\operatorname{set}^{5}$. Denote by $\mathbf{v}_{\mathbf{i}}^{(\pi)}$ and $\mathbf{p}^{(\pi)}$ the

\footnotetext{
${ }^{5}$ Let $A$ be a set containing $n$ distinct elements. Then, a permutation is simply a bijection from $A$ to itself; loosely speaking, it is a rule that changes the order of the elements of the set. For example, consider the set $A=\{a, b, c, d\}$. The following function $\pi: A \mapsto A$ is a permutation: $\pi(a)=b, \pi(b)=d, \pi(c)=c, \pi(d)=a$. Notice that $\pi$ has one fixed point $(c, c)$. If we write compactly (though a little improperly) the function $\pi$ as: $(a, b, c, d) \stackrel{\pi}{\longmapsto}(b, d, c, a)$, then it is clear why we say that a permutation reshuffles the elements of the set $A$. Very often, a permutation is defined as a bijection from a set of indexes $\mathcal{N}$ to itself. Then, for a general list of elements, we can attach to each element an index and define a permutation on the list of elements as the new list arising from a permutation of their indexes. For the previous example, we can think of the permutation $\pi$ as the following bijection form the set $\mathcal{N}=\{1,2,3,4\}$ to itself: $\pi(1)=4, \pi(2)=1, \pi(3)=3, \pi(4)=2$. In words, $\pi$ moves the element of position 1 (which was $a$ in the set $A$ ) into position 4 , the element of position 2 (which was $b$ ) into position 1 , and so on. The definition of permutation in terms of indexes fits perfectly to the case in which the list of elements we intend to permute contains elements that are repeated. Notice that, if we have a list of $n$ distinct elements, there are $n$ ! different possible permutations (thus, in the previous example $4 !=24$ different permutations can be generated). However, when some elements in the list are repeated, then the number of different possible permutations is smaller: in particular, if the list contains $n$ elements and one element is repeated $n_{1}$ times, another element is repeated $n_{2}$ times and so on, then the number of different permutations from $A$ to itself is $\left(\begin{array}{c}n \\ n_{1}, n_{2}, \ldots, n_{k}\end{array}\right)=\frac{n !}{n_{1} ! n_{2} ! \cdots n_{k} !}$, where $k$ is the number of distinct elements in A.
} 
vector of valuations and the vector of decisions obtained by permuting $\mathbf{v}_{\mathbf{i}}$ and $\mathbf{p}$ according to $\pi$. Then, the mechanism $(\mathbf{p}, \mathbf{t})$ is label free if, for all permutations $\pi: \mathcal{N} \mapsto \mathcal{N}$,

$\mathbf{p}^{(\pi)}\left(\mathbf{v}_{\mathbf{1}}^{(\pi)} ; \mathbf{v}_{\mathbf{2}}^{(\pi)}\right)=\mathbf{p}\left(\mathbf{v}_{\mathbf{1}} ; \mathbf{v}_{\mathbf{2}}\right), \quad \mathbf{t}\left(\mathbf{v}_{\mathbf{1}}^{(\pi)} ; \mathbf{v}_{\mathbf{2}}^{(\pi)}\right)=\mathbf{t}\left(\mathbf{v}_{\mathbf{1}} ; \mathbf{v}_{\mathbf{2}}\right), \quad \forall \mathbf{v}_{\mathbf{1}}, \mathbf{v}_{\mathbf{2}} \in V^{n}$

An immediate consequence of anonymity is that we can restrict our attention to a single agent. Moreover, label free implies that:

(i) the decision function can be reduced to the following single-valued function:

$$
p:\{0,1, \ldots, n\}^{2} \times V^{2} \rightarrow[0,1],
$$

that we will denote compactly by $p(k|i ; j| l)$, where $k, j \in\{0,1, \ldots, n\}$ and $i, l \in V$. This function tells us the probability of building any public good when one agent reports a low valuation $\underline{v}$ on $k$ goods (and a high valuation $\bar{v}$ on the remaining $n-k$ goods) and a valuation $i$ on that particular good, while the other agent reports a low valuation $\underline{v}$ on $j$ goods and a valuation $l$ on that particular good. Notice that, by anonymity, we have

$$
p(k|i ; j| l)=p(j|l ; k| i) .
$$

Notice also that, if $i=\underline{v}$, then $k$ cannot be equal to 0 and, if $i=\bar{v}$, then $k$ cannot be equal to $n$ (the same for $l$ and $j$ ). We overcome this problem by setting $p(0|\underline{v} ; j| l) \equiv p(n|\bar{v} ; j| l) \equiv p(k|i ; 0| \underline{v}) \equiv p(k|i ; n| \bar{v}) \equiv 0$;

(ii) similarly, the transfer function can be reduced to the following singlevalued function:

$$
t:\{0,1, \ldots, n\}^{2} \rightarrow \mathbb{R},
$$

which we will denote $t(k ; j)$. This function gives us the transfer to an agent when she reports a low valuation $\underline{v}$ on $k$ goods, while the other agent reports a low valuation $\underline{v}$ on $j \operatorname{goods}{ }^{6}$.

The linking mechanism will thus be defined in terms of $(p, t)$ as defined above.

Notice that the number of $\underline{v}$ 's contained in an agent's vector of valuations, which is crucial to our analysis, is a discrete random variable with binomial distribution with parameters $n$ and $\alpha$. In the sequel, we will denote the probability distribution of such a random variable by $f_{n, \alpha}$ and its cdf by $F_{n, \alpha}$. We thus have that the probability that an agent has a low valuation $\underline{v}$ for $k$ public goods is given by:

$$
f_{n, \alpha}(k)=\left(\begin{array}{l}
n \\
k
\end{array}\right) \alpha^{k}(1-\alpha)^{n-k} .
$$

Because the constraints the mechanism should satisfy are all interim (or ex ante) in nature, it might be useful to consider the decision and the transfer functions at the interim stage. After all, what matters for the agents, when

\footnotetext{
${ }^{6}$ Hence, thanks to anonymity and label free, types, that originally were $n$-dimensional, are now completely identified by just two elements: the number of $v$ 's contained in the agent's vector of valuations and the valuation for the public good under consideration. This latter piece of information is relevant only for the decision on providing that particular good.
} 
they play, are their interim payoffs. Following a terminology that is common in the literature, we will talk about reduced (or reduced form) decision and transfer functions. The reduced decision function will be denoted by $P_{k \mid i}$ and indicates the interim probability, from the agent viewpoint, of providing a generic public good, when the agent's reported vector of valuations contains $k \underline{v}$ 's and the (reported) valuation for that good is $i$. Formally, it is the expected value of the decision function $p(k|i ; j| l)$ where the expectation is taken with respect to the other agent's distribution of types:

$$
P_{k \mid i}=\sum_{j=0}^{n} f_{n, \alpha}(j)\left(\frac{j}{n} p(k|i ; j| \underline{v})+\frac{n-j}{n} p(k|i ; j| \bar{v})\right), k \in\{0,1, \ldots, n\}, i \in V .
$$

Likewise, we can define the reduced transfer function $T_{k}$ as the expected value of the transfer function where the expectation is taken with respect to the other agent's distribution of types:

$$
T_{k}=\sum_{j=0}^{n} f_{n, \alpha}(j) t(k ; j), \quad k \in\{0,1, \ldots, n\} .
$$

In terms of reduced form decision and transfer functions (2) and (3), the interim utility of an agent who has a low valuation for $k$ public goods and reports truthfully is thus given by:

$$
U_{k}=k \underline{v} P_{k \mid \underline{v}}+(n-k) \bar{v} P_{k \mid \bar{v}}+T_{k}, \quad k \in\{0,1, \ldots, n\} .
$$

It is worth stressing that (2) and (3) are just a more compact representation of the real mechanism, which is the one in non-reduced form $(p, t)$. However, when we work with the reduced form decisions, we must be aware that they cannot be treated as free variables: in fact, due to the assumption of anonymity, $p(k|i ; j| l)$ enters both in $P_{k \mid i}$ and in $P_{j \mid l}$ (see (2)) and thus these two probabilities cannot be chosen independently. Therefore, when choosing the optimal decision, we must go back to the non-reduced form.

\subsection{Linking vs. separate provision}

In a linking mechanism, the decision on each public good is a function of the valuations of the agents for all the $n$ public goods involved. Moreover, a single transfer, which also depends on the valuations of the agents for all the $n$ public goods, is determined.

A special case of linking mechanism is what we call a separate mechanism: consider the problem of providing a single public good $(n=1)$. Let $\pi(i ; l)$ and $\theta(i ; l)$ denote the decision and transfer functions (and $\Pi(i)$ and $\Theta(i)$ the corresponding reduced form). We will talk about separate provision of $n$ public goods and about separate mechanism, when the single good mechanism $(\pi, \theta)$ is simply replicated $n$ times. Thus, with separate provision, we have $P_{k \mid i}=\Pi(i)$, for all $k$, i.e. the reduced form decision on producing any public good depends only on the valuation of the agent for that public good; and $T_{k}=k \Theta(\underline{v})+(n-$ $k) \Theta(\bar{v})$, i.e. reduced form transfers are obtained by multiplying the number of goods for which the agent has a low (high) valuation by the separate mechanism transfer of an agent with low (high) valuation and summing them up. 


\section{Setup of the problem}

The direct revelation mechanism $(p, t)$ we look for has to be incentive feasible, i.e. Bayesian incentive compatible, interim individually rational and ex ante budget balanced. Let us state explicitly what these constraints entail in our model.

\section{Incentive Compatibility}

A direct revelation mechanism is Bayesian incentive compatible (IC) if truthtelling is a Bayesian equilibrium of the game induced by the mechanism. Thanks to anonymity and label free, the number of IC constraints of the original prob$1 \mathrm{em}^{7}$ can be greatly reduced.

According to the type of lie involved, the IC constraints can be grouped into three different classes: downward, upward and flat constraints ${ }^{8}$.

Suppose that the agent's true vector of valuations is $\mathbf{v}$ and consider the vector $\hat{\mathbf{v}}$ which is obtained from $\mathbf{v}$ by replacing some $\bar{v}$ 's with the same number of $\underline{v}$ 's. Downward IC constraints prescribe that reporting $\hat{\mathbf{v}}$ when the true vector of valuations is $\mathbf{v}$ should not be profitable to the agent ${ }^{9}$. The set of downward IC constraints is given by

$$
U_{k} \geq U_{k+i}+i(\bar{v}-\underline{v}) P_{k+i \mid \underline{v}}, k=0, \ldots, n-1, i=1, \ldots, n-k
$$

where $i$ is the number of goods on which the agent is reporting $\underline{v}$ instead of $\bar{v}$.

Of particular interest is the subgroup of downward IC constraints in which $i=1$. We call them local downward IC constraints:

$$
U_{k} \geq U_{k+1}+(\bar{v}-\underline{v}) P_{k+1 \mid \underline{v}}, \quad k=0, \ldots, n-1 .
$$

We will denote the $k$-th downward IC constraint with $\mathrm{IC}_{k}^{d}$.

Suppose that the agent's true vector of valuations is $\mathbf{v}$ and consider the vector $\hat{\mathbf{v}}$ which is obtained from $\mathbf{v}$ by replacing some $\underline{v}$ 's with the same number of $\bar{v}$ 's. Upward IC constraints prescribe that reporting $\hat{\mathbf{v}}$ when the true vector of valuations is $\mathbf{v}$ should not be profitable to the agent. The set of upward IC constraints is written compactly as

$$
U_{k} \geq U_{k-i}-i(\bar{v}-\underline{v}) P_{k-i \mid \bar{v}}, k=1, \ldots, n, i=1, \ldots, k
$$

where $i$ is the number of goods on which the agent is reporting $\bar{v}$ instead of $\underline{v}$.

Notice that combining downward and upward IC constraints, we obtain the monotonicity condition: $P_{k \mid \bar{v}} \geq P_{k+i \mid \underline{v}}, k=0, \ldots, n-1, i=1, \ldots, n-k$.

Suppose that the agent's true vector of valuations is $\mathbf{v}$ and consider the vector $\hat{\mathbf{v}}$ which is a permutation of $\mathbf{v}$; in other words, $\hat{\mathbf{v}}$ is obtained from $\mathbf{v}$ by simply interchanging some $\underline{v}$ 's with $\bar{v}$ 's. Flat IC constraints prescribe that

\footnotetext{
${ }^{7}$ In the original formulation of the problem, each of the two agents has $2^{n+1}$ possible types. Each of these types can lie in $2^{n}$ possible ways. Hence, there are $\left(2 \cdot 2^{(n+1)} \cdot 2^{n}\right)$ IC constraints.

${ }^{8}$ For a detailed discussion on downward, upward and flat IC constraints and for their derivation, see the Appendix.

${ }^{9}$ In $\hat{\mathbf{v}}$, the agent is reporting a lower average valuation for the whole bundle of goods than the truth. That's why we talk about downward IC constraints.
} 
reporting $\hat{\mathbf{v}}$ when the true vector of valuations is $\mathbf{v}$ should not be profitable to the agent. The set of flat IC constraints is given by $U_{k} \geq U_{k}+\iota(\bar{v}-\underline{v})\left(P_{k \mid \underline{v}}-P_{k \mid \bar{v}}\right)$, $k=1, \ldots, n-1, \iota=1, \ldots, \min \{k, n-k\}$, where $\iota$ is the number of interchanges, or simply

$$
P_{k \mid \bar{v}} \geq P_{k \mid \underline{v}}, \quad k=1, \ldots, n-1 .
$$

This tells us that the interim probability, from the agent viewpoint, of building a good for which the agent has a high valuation must be weakly greater than the interim probability of building a good for which the agent has a low valuation, keeping fixed the total number of $\underline{v}$ and $\bar{v}$ in $\mathbf{v}^{10}$.

\section{Individual Rationality}

The mechanism is interim individually rational (IR) if the interim utility the agent gets by participating in the mechanism is always nonnegative, i.e. $U_{k} \geq$ 0 for all $k=0, \ldots, n$. It is easy to show that the only relevant individual rationality constraint is

$$
U_{n} \geq 0 .
$$

which will be denoted by $\mathrm{IR}_{n}$. In fact, for $k=0, \ldots, n-1, \mathrm{IR}_{k}$ is necessarily true provided that the corresponding local downward IC constraint holds.

\section{Budget Balance}

The mechanism is ex-ante budget balanced (BB) if the sum of expected transfers to the agents covers the expected cost of the implemented projects, i.e.

$$
-\sum_{k=0}^{n} f_{n, \alpha}(k)\left[2 T_{k}+c\left(k P_{k \mid \underline{v}}+(n-k) P_{k \mid \bar{v}}\right)\right] \geq 0
$$

\subsection{Efficient provision}

Suppose that an incentive feasible and efficient mechanism for the provision of a single public good does exist. Then, it is quite obvious that also $n$ public goods can be efficiently provided in an incentive feasible way: we might simply use the separate mechanism that replicates the single-good efficient mechanism for each of the $n$ public goods.

It is perhaps less obvious that the converse is also true: if a linking mechanism that efficiently provides $n$ public goods in an incentive feasible way does exist, then there must exist also an efficient, incentive feasible single good mechanism.

This follows directly from the definition of efficiency and from the linear structure of the problem. Ex post efficiency prescribes that each public good is to be provided if and only if its cost is no greater than the total benefit that such good generates for the agents. This means that the efficient decision must be a function of the valuations of the agents for that particular good only. In other

\footnotetext{
${ }^{10}$ There are also IC constraints that are, in some sense, a mixture of downward (or upward) and flat constraints. However, they turn out to be irrelevant in terms of incentives (see Appendix).
} 
words, when efficiency is required, the decision function of the linking mechanism coincides with the decision function of the single-good mechanism. Besides, by incentive compatibility and because of linearity of payoffs, the reduced transfer function is largely determined by the reduced decision function. Hence, given that the decision functions coincide, transfers in the linking and in the singlegood mechanism have the same shape.

Formally, efficiency requires that

$$
P_{k \mid \underline{v}}=\Pi(\underline{v}), \quad P_{k \mid \bar{v}}=\Pi(\bar{v}) .
$$

where $\Pi$ is the efficient provision rule.

By (Upward and Downward) Incentive Compatibility (conditions (7) and (5)) and using (11), reduced transfers in the linking mechanism must be such that, for $k=0, \ldots, n-1, j=1, \ldots, n-k$,

$$
\underline{v}(\Pi(\bar{v})-\Pi(\underline{v})) \leq \frac{T_{k+j}-T_{k}}{j} \leq \bar{v}(\Pi(\bar{v})-\Pi(\underline{v}))
$$

Now, for $k=0, j=n$, the above inequality reads

$$
\underline{v}(\Pi(\bar{v})-\Pi(\underline{v})) \leq \Theta(\underline{v})-\Theta(\bar{v}) \leq \bar{v}(\Pi(\bar{v})-\Pi(\underline{v}))
$$

which is exactly Incentive Compatibility for the single-good mechanism $(\pi, \theta)$ under (11) and after setting $\Theta(\underline{v})=T_{n} / n$ and $\Theta(\bar{v})=T_{0} / n$.

Under (11), Individual Rationality in the linking mechanism requires that $T_{n} / n \geq-\underline{v} \Pi(\underline{v})$ (see condition (9)), which coincides with Individual Rationality of the low valuation type in the single-good mechanism $(\pi, \theta)$ when we set $\Theta(\underline{v})=T_{n} / n$ (the other IR constraint is redundant).

As far as budget balance is concerned, suppose that $\left(T_{0}, T_{1}, \ldots, T_{n}\right)$ (together with (11)) satisfy IC, IR and BB. Now consider the lowest possible transfers compatible with IC and IR $\left(\tilde{T}_{0}, \tilde{T}_{1}, \ldots, \tilde{T}_{n}\right)$ :

$$
\tilde{T}_{k}=-n \underline{v} \Pi(\underline{v})-(n-k)(\Pi(\bar{v})-\Pi(\underline{v})), \quad k=0, \ldots, n .
$$

Clearly, since $\left(T_{0}, T_{1}, \ldots, T_{n}\right)$ satisfies $\mathrm{BB}$, also $\left(\tilde{T}_{0}, \tilde{T}_{1}, \ldots, \tilde{T}_{n}\right)$ does. With these transfers (using (11)), budget balance (10) becomes:

$$
2[\underline{v} \Pi(\underline{v})+(1-\alpha) \bar{v}(\Pi(\bar{v})-\Pi(\underline{v}))] \geq[\alpha \Pi(\underline{v})+(1-\alpha) \Pi(\bar{v})] c
$$

which coincides with $\mathrm{BB}$ for the single-good mechanism $(\pi, \theta)$ under $(11)$ and after setting $\Theta(\underline{v})=T_{n} / n$ and $\Theta(\bar{v})=T_{0} / n$.

We summarize this observation in the following:

FACT 1: There exists an ex post efficient, incentive feasible linking mechanism for the provision of $n$ public goods if and only if there exists an ex post efficient, incentive feasible single-good mechanism.

Whether efficient provision is implementable or not depends crucially on the cost of the public good $c$. One can show that an efficient, incentive feasible mechanism to provide a single public good (and thus an efficient, incentive feasible linking mechanism to provide $n$ goods) exists if and only if $c \leq 2(\alpha \bar{v}+$ $\underline{v}) /(1+\alpha)$ or $c \geq \bar{v}+\underline{v}$. 
In particular, abstracting away from the trivial cases $c \leq 2 \underline{v}$ and $c \geq 2 \bar{v}$, we have that, for $\bar{v}+\underline{v} \leq c<2 \bar{v}$, the ex post efficient decision function

$$
\pi(\bar{v} ; \bar{v})=1, \pi(\underline{v} ; \bar{v})=\pi(\bar{v} ; \underline{v})=\pi(\underline{v} ; \underline{v})=0
$$

can be implemented in an incentive feasible way through the following transfer function:

$$
\theta(\bar{v} ; \bar{v})=-c / 2, \theta(\underline{v} ; \underline{v})=\theta(\underline{v} ; \bar{v})=\theta(\bar{v} ; \underline{v})=0
$$

For $2 \underline{v}<c \leq 2(\alpha \bar{v}+\underline{v}) /(1+\alpha)$, instead, the ex post efficient decision function

$$
\pi(\bar{v} ; \bar{v})=\pi(\underline{v} ; \bar{v})=\pi(\bar{v} ; \underline{v})=1, \pi(\underline{v} ; \underline{v})=0 .
$$

can be implemented in an incentive feasible way through the following transfers:

$$
\theta(\bar{v} ; \bar{v})=-c / 2, \theta(\underline{v} ; \underline{v})=0, \theta(\underline{v} ; \bar{v})=-\underline{v}, \theta(\bar{v} ; \underline{v})=\underline{v}-c .
$$

\section{Optimal provision}

The interesting case is when efficient provision of a single public good is not achievable, i.e.

$$
\underline{c} \equiv 2(\alpha \bar{v}+\underline{v}) /(1+\alpha)<c<\bar{v}+\underline{v} \equiv \bar{c} .
$$

Clearly, in this case an efficient, incentive feasible linking mechanism does not exist either; however, we might wonder whether linking might help reduce inefficiency with respect to separate provision.

\subsection{The optimal separate mechanism}

Under (12), the incentive feasible single-good mechanism that maximizes expected total welfare (i.e. the optimal single-good mechanism) has the following decision function:

$$
\begin{gathered}
\pi(\bar{v} ; \bar{v})=1, \quad \pi(\underline{v} ; \underline{v})=0, \\
\pi(\bar{v} ; \underline{v})=\pi(\underline{v} ; \bar{v})=1-\frac{(1+\alpha)(c-\underline{c})}{2[(1-\alpha)(\bar{v}-\underline{v})-\alpha(\bar{v}+\underline{v}-c)]} \equiv \hat{\pi} .
\end{gathered}
$$

The optimal separate mechanism is then an $n$-replica of this optimal singlegood mechanism.

Notice that this mechanism differs from the efficient one only in that, when the valuations of the two agents are mixed (one agent has a low valuation, the other has a high valuation), the good is provided with probability less than one. As $c$ approaches $\underline{c}$, we get closer and closer to efficiency. The transfer function is not determined uniquely. However, one transfer function that does the job is the following:

$$
\theta(\underline{v} ; \underline{v})=0, \quad \theta(\underline{v} ; \bar{v})=-\hat{\pi} \underline{v}, \quad \theta(\bar{v} ; \underline{v})=\hat{\pi}(\underline{v}-c), \quad \theta(\bar{v} ; \bar{v})=-c / 2 .
$$

By setting $\theta(\underline{v} ; \bar{v})=\underline{v}$ and $\theta(\bar{v} ; \underline{v})=(\underline{v}-c)$ if the good is provided and 0 otherwise, we obtain a mechanism that is also ex post budget balanced and ex post individually rational. 
Total expected welfare generated by the optimal separate mechanism is given by :

$$
W=(1-\alpha)[2 \alpha \hat{\pi}(\bar{v}+\underline{v}-c)+(1-\alpha)(2 \bar{v}-c)]
$$

which, compared to the potential welfare $W^{*}$, gives:

$$
\frac{W}{W^{*}}=1-\frac{2 \alpha(\bar{v}+\underline{v}-c)}{2 \alpha(\bar{v}+\underline{v}-c)+(1-\alpha)(2 \bar{v}-c)}(1-\hat{\pi}) .
$$

\subsection{The optimal linking mechanism}

In this section, we derive the optimal linking mechanism, i.e. the mechanism that maximizes expected total welfare. In order to do so, we will adopt the following strategy that dates back to Myerson (1981): we first solve the reduced optimization problem where only the local downward IC constraints are considered (plus, of course, IR and BB) ${ }^{11}$.

The reduced optimization problem can be formulated as follows:

$$
\max _{(p, t)} 2 \sum_{k=0}^{n} f_{n, \alpha}(k) U_{k}
$$

subject to:

- $\operatorname{IR}_{n}$ (condition (9)),

- $\mathrm{IC}_{k}^{d}, k=0, \ldots, n-1$ (conditions $\left.(6)\right)$,

- BB (condition (10)),

- and the feasibility conditions $p(k|i ; j| l) \in[0,1]$.

After we find a tentative solution to this reduced problem, we verify ex-post that this solution also satisfies the remaining IC constraints.

The following proposition characterizes the optimal linking mechanism:

Proposition 1: Suppose that $\underline{c}<c<\bar{c}$. Let $\bar{k}$ be the highest integer such that

$$
\frac{2 \bar{k} F_{n, \alpha}(\bar{k})}{n(1-\alpha)+2 n \alpha F_{n-1, \alpha}(\bar{k}-1)} \leq 1+\frac{\bar{c}-c}{\bar{v}-\underline{v}} .
$$

Then the following linking mechanism is incentive feasible and maximizes the total expected welfare:

- $p(k|\bar{v} ; j| \bar{v})=1, \quad k, j=0, \ldots, n-1$;

- $p(k|\underline{v} ; j| \underline{v})=0, \quad k, j=1, \ldots, n$;

- $p(k|\underline{v} ; j| \bar{v})=p(j|\bar{v} ; k| \underline{v})=1, \quad k \leq \bar{k}, \quad j=0, \ldots, n-1$;

- $p(k|\underline{v} ; j| \bar{v})=p(j|\bar{v} ; k| \underline{v})=0, \quad k \geq \bar{k}+2, \quad j=0, \ldots, n-1$;

\footnotetext{
${ }^{11}$ Here we are using the attribute reduced to mean that some constraints are ignored. This has nothing to do with the concept of reduced form probabilities and transfers introduced before.
} 
- $p(\bar{k}+1|\underline{v} ; j| \bar{v})=p(j|\bar{v} ; \bar{k}+1| \underline{v})$ s.t. $P_{\bar{k}+1 \mid \underline{v}}=(1-\alpha) \gamma, \quad j=0, \ldots, n-1$;

- $T_{0}=-(1-\alpha)^{-n} \sum_{k=1}^{n} f_{n, \alpha}(k)\left(T_{k}+\frac{c}{2}\left(k P_{k \mid \underline{v}}+(n-k) P_{k \mid \bar{v}}\right)\right)-n \frac{c}{2} P_{0 \mid \bar{v}}$;

- $T_{k}=(\bar{v}-\underline{v}) \sum_{j=k+1}^{n} P_{j \mid \underline{v}}-k \underline{v} P_{k \mid \underline{v}}-(n-k) \bar{v} P_{k \mid \bar{v}}, \quad k=1, \ldots, n-1$;

- $T_{n}=-n \underline{v} P_{n \mid \underline{v}}$

where

$\gamma=\frac{2(\bar{v}+\underline{v}-c) \sum_{k=1}^{\bar{k}} k f_{n, \alpha}(k)-2(\bar{v}-\underline{v}) \sum_{k=1}^{\bar{k}} F_{n, \alpha}(k-1)+n(1-\alpha)(2 \bar{v}-c)}{2(\bar{v}-\underline{v}) F_{n, \alpha}(\bar{k})-2(\bar{v}+\underline{v}-c)(\bar{k}+1) f_{n, \alpha}(\bar{k}+1)}$.

The structure of the proof, which is relegated in the Appendix, is the following: first we show that, in the reduced optimization problem, $\mathrm{IR}_{n}, \mathrm{BB}, \mathrm{IC}_{k}^{d}$ with $k>0$ must be binding in an optimum. This allows us to determine the optimal reduced form transfers and to simplify the optimization problem. We then solve the reduced optimization problem under the remaining constraints ( $\mathrm{IC}_{0}^{d}$ and the feasibility conditions). In particular, we characterize the shape of the optimal decision function by means of the Kuhn-Tucker conditions. Then we completely characterize the optimal decision function by determining the optimal cutoff value $\bar{k}$; this is done by using the fact that also $\mathrm{IC}_{0}^{d}$ will bind in the optimum. Finally, we go back to the original optimization problem showing that the solution to the reduced optimization problem satisfies also the IC constraints that were initially neglected.

\section{Discussion}

The optimal decision function prescribes that, when both agents have a high valuation for a good, then this good will be provided for sure. When, instead, both have a low valuation for a good, then this will not be produced. So far, the optimal decision function coincides with the efficient one and also with the optimal separate mechanism.

Just like in the optimal separate mechanism, inefficiency arises when agents have mixed valuations for the public good, i.e. one agent has a high valuation, the other a low valuation.

In the optimal separate mechanism, when agents have mixed valuations, the good is provided with probability less than one.

In the optimal linking mechanism, instead, when agents have mixed valuations typically the good is either built for sure or not built at all. This "zero-one" decision is determined by looking at the valuation for the entire bundle of goods, which is exactly the spirit of linking. In particular, a good will be provided if the agent with a low valuation for that particular good has a "high" average valuation for the whole bundle of goods, will not built otherwise. Therefore, only the agent with a low valuation for the good is "pivotal". There is just one case in which the decision does not take a "zero-one" form and this is when the low valuation agent has a low valuation for exactly $\bar{k}+1$ goods $^{12}$. In this case,

\footnotetext{
${ }^{12}$ And $\gamma>0$.
} 
Proposition 1 identifies only the reduced form probability of building the good $P_{\bar{k}+1 \mid \underline{v}}=(1-\alpha) \gamma$. There are of course infinite non reduced form probabilities $p_{\bar{k}+1|\underline{v} ; j| \bar{v}}$ that are consistent with this reduced form probability. One could for example choose a constant $p(\bar{k}+1|\underline{v} ; j| \bar{v})=\gamma$.

The optimal linking mechanism identifies unambiguously only the reduced form transfers.

$$
\begin{gathered}
T_{n}=-n \underline{v} P_{n \mid \underline{v}} \\
T_{k}=(\bar{v}-\underline{v}) \sum_{j=k+1}^{n} P_{j \mid \underline{v}}-k \underline{v} P_{k \mid \underline{v}}-(n-k) \bar{v} P_{k \mid \bar{v}}, \quad k=1, \ldots, n-1 .
\end{gathered}
$$

Transfers extract all the surplus from those agents with $\bar{k}<k \leq n$. Instead, agent with $k<\bar{k}$ will enjoy a strictly positive net surplus $(\bar{v}-\underline{v})[(1-\alpha)(\bar{k}-$ $k)+P_{\bar{k}+1 \mid \underline{v}}$, that will be larger the lower is $k$. An agent with $k=\bar{k}$ will have a positive or null net surplus depending on whether $\gamma$ is positive or null.

Basically, transfers distribute the expected net welfare only to those agents that would have liked to have more than $\bar{k}$ goods provided. Among these agents, those that wanted to have more public goods built will get a higher transfer. Instead transfers leave no rent to those agents for which less than $\bar{k}$ goods are worth building.

The expected transfer to the agent with a high valuation for all the goods is designed as to balance the budget.

\subsection{Intuition}

The optimal linking mechanism just described works in a quite different way compared with the optimal separate mechanism. Typically, it is the high valuation agent the one that is tempted to free ride. He has an incentive to pretend to have a low valuation hoping to meet a high valuation opponent in order to have the good provided anyway, without having to bear the cost. Because budget balance and individual rationality constraints impose tight upper and lower bounds to the admissible transfers, the only way to eliminate the incentive to free ride is to introduce some inefficient decisions.

In the optimal separate mechanism, this is done through a reduction in the probability of producing the public good when agents have mixed valuations.

The possibility of linking the decisions on different public goods gives rise to a completely different solution to the free riding problem. Instead of reducing a little the probability of providing the good when agents have mixed valuations, in the optimal linking mechanism the decision is more drastic: in general, a the good will either be produced for sure or not at all. This decision is conditioned on the average valuation of the low valuation agent for the whole vector of goods.

To make things easier, suppose $\gamma=0$. Consider an agent with a low average valuation for the bundle of public goods, namely an agent with $k>\bar{k}$, and suppose this agent is considering reporting a low valuation for a good for which he has a high valuation. By doing so, the agent would suffer a drastic drop in the probability of having the good built from $P_{k \mid \bar{v}}$ to zero. This drop is even more pronounced for the "cutoff" agent (i.e. the agent with a low valuation for exactly $\bar{k}$ goods). By falsely reporting $\underline{v}$ for a good for which his true valuation is $\bar{v}$, not only the probability of producing that good would fall from $P_{k \mid \bar{v}}$ to zero, 
but also the probability of producing any other good for which his valuation is low would go to zero. It should thus be clear that agents with a low average valuation for the bundle of goods will have no incentive to free ride. This is of course inefficient; however, this allows to ask higher payments from these agents and hence to alleviate the budget balance problem. In fact, this budget surplus is used to finance the deficit produced by the agents with a high average valuation for the bundle (namely, those agents with $k<\bar{k}$ ). In fact, if one of these agent falsely reported a low valuation for some good, he would experience only a little reduction in the probability of having the good provided. This would be profitable if the reduction in the due payment were substantial. However, using the budget surplus collected from the low average valuation types, payments requested from high average valuation types are reduced enough to make free riding non profitable.

\subsection{Cutoff value}

The decision on those goods for which agents have mixed valuations is determined by the cutoff value $\bar{k}$. This number is defined implicitly by inequality (13). In general, it depends on the values of all the parameters involved, so we have $\bar{k}=\bar{k}(c, \bar{v}, \underline{v}, \alpha, n)$. We know that $\bar{k}<n$ (otherwise we would have efficiency, but we know this is not possible).

First, we provide, in the following lemma, a lower bound to $\bar{k}$.

Lemma 1: Let $\bar{k}$ be the highest integer that satisfies (13). Then, for all $\bar{v}, \underline{v}$ and for all c satisfying (12),

$$
\bar{k} \geq\lfloor n \alpha\rfloor .
$$

Proof. For (13) to be satisfied for all $\bar{v}, \underline{v}$ and for all $c$ satisfying (12), we must have

$$
\frac{2 \bar{k} F_{n, \alpha}(\bar{k})}{n(1-\alpha)+2 n \alpha F_{n-1, \alpha}(\bar{k}-1)} \leq 1
$$

or

$$
2 \bar{k} F_{n, \alpha}(\bar{k}) \leq n(1-\alpha)+2 n \alpha F_{n-1, \alpha}(\bar{k}-1) \leq 1 .
$$

Subtracting from both sides $2 n \alpha F_{n, \alpha}(\bar{k})$, using the fact that $F_{n, \alpha}(x)-F_{n-1, \alpha}(x-$ $1)=(1-\alpha) f_{n-1, \alpha}(x)$ and rearranging, we get

$$
2 n \alpha(1-\alpha) f_{n-1, \alpha}(\bar{k}) \leq n(1-\alpha)+2(n \alpha-\bar{k}) F_{n, \alpha}(\bar{k}) .
$$

Now, $n(1-\alpha) f_{n-1, \alpha}(x)=(n-x) f_{n-1, \alpha}(x)$. Substituting in the last inequality, we obtain

$$
2 \alpha(n-\bar{k}) f_{n, \alpha}(\bar{k}) \leq n(1-\alpha)+2(n \alpha-\bar{k}) F_{n, \alpha}(\bar{k}) .
$$

Because $f_{n, \alpha}(x) \leq F_{n, \alpha}(x)$, it is sufficient to show that

$$
2 \alpha(n-\bar{k}) f_{n, \alpha}(\bar{k}) \leq n(1-\alpha)+2(n \alpha-\bar{k}) f_{n, \alpha}(\bar{k}),
$$

or

$$
2 \bar{k} f_{n, \alpha}(\bar{k}) \leq n
$$


Finally, using the fact that $f_{n, \alpha}(x)=\frac{n}{x} \alpha f_{n-1, \alpha}(x-1)$, we get

$$
2 \alpha f_{n-1, \alpha}(\bar{k}-1) \leq 1,
$$

which is certainly true for $\bar{k}=\lfloor n \alpha\rfloor$. In fact, since $f_{n-1, \alpha}(x)$ is maximized for $x=\lfloor n \alpha\rfloor$, it must be $f_{n-1, \alpha}(\lfloor n \alpha\rfloor-1) \leq \frac{1}{2}$.

Second, we characterize the value of $\bar{k}$ when the number of public goods $n$ grows to infinity.

Lemma 2: Let $\bar{k}$ be the highest integer that satisfies (13). Then

$$
\lim _{n \rightarrow \infty} \frac{\bar{k}}{n}=\frac{1+\alpha}{2}\left(1+\frac{\bar{c}-c}{\bar{v}-\underline{v}}\right) .
$$

For the sake of the proof, it is convenient to set $L \equiv \frac{1+\alpha}{2}\left(1+\frac{\bar{c}-c}{\bar{v}-\underline{v}}\right)$ and rewrite (13) as

$$
\frac{\frac{\bar{k}}{n} F_{n, \alpha}(\bar{k})}{1+\frac{2 \alpha}{1+\alpha}\left(F_{n-1, \alpha}(\bar{k}-1)-1\right)} \leq \frac{1+\alpha}{2}\left(1+\frac{\bar{c}-c}{\bar{v}-\underline{v}}\right) \equiv L .
$$

Notice that $\alpha<L<1$.

The proof is based on a large number argument: by the Strong Law of Large Numbers, if $\left(X_{n}\right)_{n \geq 1}$ is a sequence of random variables with binomial distribution $F_{n, \alpha}$, then $\frac{X_{n}}{n} \stackrel{P}{\rightarrow} \alpha$, i.e.

$$
\forall \epsilon>0, \forall \delta>0, \exists N_{\epsilon, \delta}: \operatorname{Prob}\left(\left|\frac{X_{n}}{n}-\alpha\right|>\epsilon\right)<\delta, \forall n>N_{\epsilon, \delta} .
$$

Proof. The proof goes by contradiction: suppose that (14) is false, i.e. suppose that: $\exists \epsilon^{\prime}>0: \forall N,\left|\frac{\bar{k}}{n}-L\right| \geq \epsilon^{\prime}$, for some $n>N$. We distinguish two cases: (i) $\frac{\bar{k}}{n} \geq L+\epsilon^{\prime}$ and (ii) $\frac{\bar{k}}{n} \leq L-\epsilon$.

(i) $\exists \epsilon^{\prime}>0: \forall N, \frac{\bar{k}}{n} \geq L+\epsilon^{\prime}$, for some $n>N$. Take such $\epsilon^{\prime}$. In (16), set $\epsilon=\epsilon^{\prime}, \delta=\epsilon^{\prime} /\left(1+\epsilon^{\prime}\right)$. Take $N=N_{\epsilon, \delta}$ and consider $n>N$ for which $\frac{\bar{k}}{n} \geq L+\epsilon^{\prime}$. We have the following chain of inequalities:

$$
\begin{gathered}
1-\delta<\operatorname{Prob}\left(\alpha-\epsilon^{\prime} \leq \frac{X_{n}}{n} \leq \alpha+\epsilon^{\prime}\right) \leq \operatorname{Prob}\left(\frac{X_{n}}{n} \leq \alpha+\epsilon^{\prime}\right) \leq \\
\leq \operatorname{Prob}\left(\frac{X_{n}}{n} \leq L+\epsilon^{\prime}\right) \leq \operatorname{Prob}\left(\frac{X_{n}}{n} \leq \frac{\bar{k}}{n}\right)=F_{n, \alpha}(\bar{k}),
\end{gathered}
$$

where the third inequality follows from the fact that $L>\alpha$ and the fourth from the assumption $\frac{\bar{k}}{n} \geq L+\epsilon^{\prime}$. We thus have:

$\frac{\frac{\bar{k}}{n} F_{n, \alpha}(\bar{k})}{1+\frac{2 \alpha}{1+\alpha}\left(F_{n-1, \alpha}(\bar{k}-1)-1\right)} \geq \frac{\bar{k}}{n} F_{n, \alpha}(\bar{k}) \geq\left(L+\epsilon^{\prime}\right) F_{n, \alpha}(\bar{k})>\left(L+\epsilon^{\prime}\right)(1-\delta)>L$,

contradicting the hypothesis that $\bar{k}$ satisfies (15) (or (13)). In the above, the first inequality follows from the fact that the denominator is strictly positive 
but no greater than 1 and the last from the definition of $\delta$ and from the fact that $L<1$.

(ii) $\exists \epsilon^{\prime}>0: \forall N, \frac{\bar{k}}{n} \leq L+\epsilon^{\prime}$, for some $n>N$. Take such $\epsilon^{\prime}$, and consider $N>1 / \epsilon^{\prime}$. Let $A$ be the set of integers that are strictly greater than $N$ and that satisfy $\frac{\bar{k}}{n} \leq L+\epsilon^{\prime}$. This set must contain infinite elements. Notice that $\epsilon^{\prime}-1 / n>0$ for all $n \in A$. Notice also that, if $\frac{\bar{k}}{n} \leq L+\epsilon^{\prime}$ for all $n \in A$, then, for all $\epsilon^{\prime \prime} \in\left(0, \epsilon^{\prime}\right)$ we also have $\frac{\bar{k}}{n} \leq L+\epsilon^{\prime \prime}$ for all $n \in A$. Thus, take $\epsilon^{\prime \prime}$ such that:

$$
\left\{\begin{array}{l}
\epsilon^{\prime \prime}>0 \\
\alpha+\epsilon^{\prime \prime} \leq \frac{\bar{k}+1}{n}, \text { for all } n \in A \\
\alpha+2 \epsilon^{\prime \prime} \leq \frac{k+1}{n-1}, \text { for all } n \in A \\
\epsilon^{\prime \prime} \leq \epsilon^{\prime}-\frac{1}{n}, \text { for all } n \in A
\end{array}\right.
$$

This system admits a solution: in fact, Lemma 1 implies that $\frac{\bar{k}+1}{n}>\alpha$, for all $n$, which, in turn, implies that $\frac{\bar{k}+1}{n-1}>\alpha$, for all $n$; besides, $\epsilon^{\prime}-1 / n>0$ for all $n \in A$. in $A$.

Finally, in (16), set $\epsilon=\epsilon^{\prime \prime}, \delta=\frac{\epsilon^{\prime \prime}}{2(2-\alpha)}$. Consider any $n>N_{\epsilon, \delta}$ that also lies

Notice that, since, for all $n \in A, \frac{\bar{k}}{n} \leq L+\epsilon^{\prime}$ and $\epsilon^{\prime \prime} \leq \epsilon^{\prime}-\frac{1}{n}$, we have that $\frac{\bar{k}+1}{n} \leq L+\epsilon^{\prime \prime}$.

We have the following chains of inequalities:

$$
\begin{gathered}
1-\delta<\operatorname{Prob}\left(\alpha-\epsilon^{\prime \prime} \leq \frac{X_{n}}{n} \leq \alpha+\epsilon^{\prime \prime}\right) \leq \operatorname{Prob}\left(\frac{X_{n}}{n} \leq \alpha+\epsilon^{\prime \prime}\right) \leq \\
\leq \operatorname{Prob}\left(\frac{X_{n}}{n} \leq \frac{\bar{k}+1}{n}\right)=F_{n, \alpha}(\bar{k}+1),
\end{gathered}
$$

where the third inequality follows from the fact that $\alpha+\epsilon^{\prime \prime} \leq \frac{\bar{k}+1}{n}$; and:

$$
\begin{gathered}
\delta>\operatorname{Prob}\left(\left|\frac{X_{n-1}}{n-1}-\alpha\right|>\epsilon\right) \geq \operatorname{Prob}\left(\frac{X_{n-1}}{n-1}>\alpha+\epsilon\right) \geq \\
\geq \operatorname{Prob}\left(\frac{X_{n-1}}{n-1}>\frac{\bar{k}+1}{n-1}-\epsilon\right) \geq \operatorname{Prob}\left(\frac{X_{n-1}}{n-1}=\frac{\bar{k}+1}{n-1}\right)=f_{n-1, \alpha}(\bar{k}+1),
\end{gathered}
$$

where the third inequality follows from the fact that $\alpha+2 \epsilon^{\prime \prime} \leq \frac{\bar{k}+1}{n-1}$. We claim that $\bar{k}$ is not the highest integer satisfying (15) (or (13)). To see this, take $\bar{k}+1$ in (15). We have:

$$
\begin{gathered}
\frac{\frac{\bar{k}+1}{n} F_{n, \alpha}(\bar{k}+1)}{1+\frac{2 \alpha}{1+\alpha}\left(F_{n-1, \alpha}(\bar{k})-1\right)}=\frac{\frac{\bar{k}+1}{n} F_{n, \alpha}(\bar{k}+1)}{1+\frac{2 \alpha}{1+\alpha}\left(F_{n, \alpha}(\bar{k}+1)-(1-\alpha) f_{n-1, \alpha}(\bar{k}+1)-1\right)}< \\
<\frac{\frac{\bar{k}+1}{n}}{1+\frac{2 \alpha}{1+\alpha} \delta(\alpha-2)}=\frac{\frac{\bar{k}+1}{n}(1+\alpha)}{1+\alpha-\alpha \epsilon^{\prime \prime}} \leq \frac{\left(L-\epsilon^{\prime \prime}\right)(1+\alpha)}{1+\alpha-\alpha \epsilon^{\prime \prime}}<L,
\end{gathered}
$$

where in the first equality we used $F_{n, \alpha}(x)-(1-\alpha) f_{n-1, \alpha}(x)=F_{n-1, \alpha}(x-1)$ and where the last inequality follows from the fact that $L<1$. 


\subsection{Asymptotic efficiency}

An immediate consequence of Lemma 2 is that the decision function of the optimal bundling mechanism converges in probability to the efficient one.

LEMMA 3: Let $p_{n}$ denote the decision function of the optimal linking mechanism with $n$ goods and $p^{*}$ the efficient decision function. Then, for any $\epsilon>0$

$$
\lim _{n \rightarrow \infty} \operatorname{Prob}\left(p^{*}-p_{n}>\epsilon\right)=0
$$

Proof. The event $\left(p^{*}-p_{n}>\epsilon\right)$ occurs only if one agent (or both) has a low valuation for more than $\bar{k}$ goods. In other words

$$
\operatorname{Prob}\left(p^{*}-p_{n}>\epsilon\right) \leq \beta^{2}+2 \beta(1-\beta)=\beta(2-\beta)
$$

where $\beta=\operatorname{Prob}\left(X_{n}>\bar{k}\right)=1-F_{n, \alpha}(\bar{k})$ is the probability that one agent has a low valuation for more than $\bar{k}$ goods. Notice that

$$
\lim _{n \rightarrow \infty} F_{n, \alpha}(\bar{k})=\lim _{n \rightarrow \infty} \operatorname{Prob}\left(\frac{X_{n}}{n} \leq \frac{\bar{k}}{n}\right)=1
$$

because $\bar{k} / n$ converges to $L>\alpha$ (Lemma 2 ) and $X_{n} / n$ converges in probability to $\alpha$ (by the Strong Law of Large Numbers). We thus have that $\beta \rightarrow 0$ as $n \rightarrow \infty$, which completes the proof.

\subsection{An illustrating example}

Suppose that the parameters of the model are the following: $\bar{v}=1, \underline{v}=0$, $c=3 / 4, \alpha=1 / 3$.

The optimal linking mechanism prescribes that, if agent have mixed valuations for any public good, then the decision on that good will depend on the value of $\bar{k}$ defined by (13), which depends on $n$.

In the following table, we report the value of $\bar{k}$ for different values of $n$. We also report the relative efficiency of the mechanism, measured by the ratio between the expected welfare generated by the mechanism and the potential welfare (i.e. the expected welfare that would be achieved by an ex-post efficient decision).

As a comparison, we also report the relative efficiency of the optimal separate mechanism and also the relative efficiency of the asymptotically efficient mechanism introduced by Fang and Norman (2006a).

Notice how linking yields a substantial reduction in inefficiency, even when only two goods are bundled. With 3 goods, the efficiency loss is already below 1\%. Notice also that, compared with the mechanism by Fang and Norman, the optimal linking mechanism performs substantially better for low values of $n$. Obviously, since both are asymptotically efficient, the difference between these two mechanisms vanishes as $n$ grows. 


\begin{tabular}{||l||c||c|c|c||}
\hline \hline$n$ & $k$ & Linking Mech. & Separate Mech. & Fang, Norman (2006a) \\
\hline \hline 2 & 1 & 0.9778 & 0.9524 & 0.9444 \\
\hline 3 & 2 & 0.9901 & 0.9524 & 0.9815 \\
\hline 4 & 3 & 0.9958 & 0.9524 & 0.9938 \\
\hline 5 & 4 & 0.9983 & 0.9524 & 0.9592 \\
\hline 6 & 4 & 0.9993 & 0.9524 & 0.9850 \\
\hline 7 & 5 & 0.9993 & 0.9524 & 0.9945 \\
\hline 8 & 6 & 0.9996 & 0.9524 & 0.9980 \\
\hline 9 & 7 & 0.9998 & 0.9524 & 0.9993 \\
\hline 10 & 8 & 0.9999 & 0.9524 & 0.9969 \\
\hline 15 & 12 & 1.0000 & 0.9524 & 0.9997 \\
\hline 20 & 16 & 1.0000 & 0.9524 & 1.0000 \\
\hline \hline
\end{tabular}

Table 1: Relative efficiency.

\section{Conclusion}

In this paper, we consider the problem of providing $n$ identical non-excludable public goods in a two-agent economy with binary valuations for each public good.

Instead of taking $n$ separate decisions, we consider the possibility of using a linking mechanism, that is a mechanism in which the decision on each public good is a function of the valuations of the agents for all public goods. Focussing on a situation in which efficiency is not achievable, we characterize the optimal incentive feasible linking mechanism.

In this mechanism the efficient decision is taken when both agents have the same valuation for a good. Instead, when agents' valuations for any good are in contrast (one agent has a high valuation, the other has a low valuation), the good is provided if and only if the low valuation agent has a sufficiently high average valuation for the whole set of public goods. Otherwise, an inefficient decision is taken: the good is not provided at all or it is provided with probability strictly less than one. The optimal linking mechanism improves upon the optimal separating mechanism because the probability that an inefficient decision is actually taken is low, and converges to zero as the number of public goods considered grows to infinity. 


\section{A IC constraints}

Downward IC CONSTRAints. Let $k(\mathbf{v})$ denote the number of $\underline{v}$ 's contained in $\mathbf{v}$ (obviously, $n-k(\mathbf{v})$ will be the number of $\bar{v}$ 's). Suppose now that the true vector of valuations is $\mathbf{v}$, with $k(\mathbf{v})<n$, and suppose that the reported vector $\hat{\mathbf{v}}$ is obtained from $\mathbf{v}$ by simply replacing some of the $\bar{v}$ 's with an equal number of $\underline{v}$ 's (as an example, take $\mathbf{v}=(\underline{v}, \underline{v}, \bar{v}, \bar{v}, \bar{v})$ and $\hat{\mathbf{v}}=(\underline{v}, \underline{v}, \bar{v}, \underline{v}, \underline{v}))$. Then, $k(\hat{\mathbf{v}})>k(\mathbf{v})$ (in the example, $k(\hat{\mathbf{v}})=4>k(\mathbf{v})=2)$. Such an agent is reporting truthfully his valuation of the $k(\mathbf{v})$ goods for which he has a low valuation $\underline{v}$. Now consider the $n-k(\mathbf{v})$ goods for which he has a high valuation $\bar{v}$ : for some of them (namely, $k(\hat{\mathbf{v}})-k(\mathbf{v})$ goods), the agent falsely reports a low valuation; for the remaining $n-k(\hat{\mathbf{v}})$ goods, he is truthfully reporting a high valuation. All in all, his interim utility is given by

$$
U(\hat{\mathbf{v}} ; \mathbf{v})=k(\mathbf{v}) \underline{v} P_{k(\hat{\mathbf{v}}) \mid \underline{v}}+(k(\hat{\mathbf{v}})-k(\mathbf{v})) \bar{v} P_{k(\hat{\mathbf{v}}) \mid \underline{v}}+(n-k(\hat{\mathbf{v}})) \bar{v} P_{k(\hat{\mathbf{v}}) \mid \bar{v}}+T_{k(\hat{\mathbf{v}})}
$$

By adding and subtracting $\left(k(\hat{\mathbf{v}}) \underline{v} P_{k(\hat{\mathbf{v}}) \mid \underline{v}}\right.$, we can simplify the above expression in

$$
U(\hat{\mathbf{v}} ; \mathbf{v})=U_{k(\hat{\mathbf{v}})}+(k(\hat{\mathbf{v}})-k(\mathbf{v}))(\bar{v}-\underline{v}) P_{k(\hat{\mathbf{v}}) \mid \underline{v}} .
$$

Incentive compatibility requires $U(\mathbf{v} ; \mathbf{v}) \geq U(\hat{\mathbf{v}} ; \mathbf{v})$, which reduces to

$$
U_{k(\mathbf{v})} \geq U_{k(\hat{\mathbf{v}})}+(k(\hat{\mathbf{v}})-k(\mathbf{v}))(\bar{v}-\underline{v}) P_{k(\hat{\mathbf{v}}) \mid \underline{v}},
$$

where $U_{k(\mathbf{v})}$ is the interim utility, under truthtelling, of an agent with vector of valuations $\mathbf{v}$ (see (4)). The set of downward IC constraints can thus be written compactly as in (5).

UpWARD IC CONSTRAints. Suppose now that the true vector of valuations is $\mathbf{v}$, with $k(\mathbf{v})>0$, and suppose that the reported vector $\hat{\mathbf{v}}$ is obtained from $\mathbf{v}$ by simply replacing some of the $\underline{v}$ 's with an equal number of $\bar{v}$ 's (as an example, take $\mathbf{v}=$ $(\underline{v}, \underline{v}, \bar{v}, \bar{v}, \bar{v})$ and $\hat{\mathbf{v}}=(\underline{v}, \bar{v}, \bar{v}, \bar{v}, \bar{v})$ ). Then, $k(\hat{\mathbf{v}})<k(\mathbf{v})$ (in the example, $k(\hat{\mathbf{v}})=1<$ $k(\mathbf{v})=2)$. By reasoning exactly in the same fashion as before, one could obtain the general form of the upward IC constraint for an agent with true vector of valuations $\mathbf{v}$ who reports $\hat{\mathbf{v}}$.

$$
U_{k(\mathbf{v})} \geq U_{k(\hat{\mathbf{v}})}-(k(\mathbf{v})-k(\hat{\mathbf{v}}))(\bar{v}-\underline{v}) P_{k(\hat{\mathbf{v}}) \mid \underline{v}} .
$$

The set of upward IC constraints can thus be written compactly as (7).

Flat IC Constraints. Suppose the agent reports a vector of valuations $\hat{\mathbf{v}}$ which is just a permutation of the true vector of valuations $\mathbf{v}$; when this happens, we have $k(\mathbf{v})=k(\hat{\mathbf{v}})$ (as an example, take $\mathbf{v}=(\underline{v}, \underline{v}, \bar{v}, \bar{v}, \bar{v})$ and $\hat{\mathbf{v}}=(\underline{v}, \bar{v}, \bar{v}, \bar{v}, \underline{v})$; in this case $k(\mathbf{v})=k(\hat{\mathbf{v}})=2)$. If we compare $\hat{\mathbf{v}}$ with the "true" vector $\mathbf{v}$ entry by entry, there will be a certain number (possibly zero) of entries that coincide (in the example, three entries coincide: the first, the third and the fourth). The remaining entries, instead, will display $\underline{v}$ 's in place of $\bar{v}$ 's, or viceversa. To go back from $\hat{\mathbf{v}}$ to $\mathbf{v}$, we thus have to interchange $\underline{v}$ 's with $\bar{v}$ 's. How many times? This depends on the number of coincident entries. If $\mathbf{v}$ and $\hat{\mathbf{v}}$ have $m$ coincident entries, then $(n-m) / 2$ interchanges will be necessary to pass from $\hat{\mathbf{v}}$ to $\mathbf{v}$. This number of interchanges is denoted $\iota(\mathbf{v}, \hat{\mathbf{v}})$.

What is the interim utility of an agent who reports $\hat{\mathbf{v}}$ instead of $\mathbf{v}$ ? The vector $\hat{\mathbf{v}}$ will have $k(\mathbf{v})-\iota(\mathbf{v}, \hat{\mathbf{v}})$ of $\underline{v}$ 's in the correct position and $n-k(\mathbf{v})-\iota(\mathbf{v}, \hat{\mathbf{v}})$ of $\bar{v}$ 's in the correct position. Instead, there will be $\iota(\mathbf{v}, \hat{\mathbf{v}})$ of $\underline{v}$ 's in place of $\bar{v}$ 's. Thus, the interim utility of the agent will be

$$
\begin{aligned}
U(\hat{\mathbf{v}} ; \mathbf{v})=( & k(\mathbf{v})-\iota(\mathbf{v}, \hat{\mathbf{v}})) \underline{v} P_{k(\hat{\mathbf{v}}) \mid \underline{v}}+(n-k(\mathbf{v})-\iota(\mathbf{v}, \hat{\mathbf{v}})) \bar{v} P_{k(\hat{\mathbf{v}}) \mid \bar{v}} \\
& +\iota(\mathbf{v}, \hat{\mathbf{v}}) \underline{v} P_{k(\hat{\mathbf{v}}) \mid \bar{v}}+\iota(\mathbf{v}, \hat{\mathbf{v}}) \bar{v} P_{k(\hat{\mathbf{v}}) \mid \underline{v}}+T_{k(\hat{\mathbf{v}}) .} .
\end{aligned}
$$


Incentive compatibility requires

$$
U_{k(\mathbf{v})} \geq U_{k(\hat{\mathbf{v}})}+\iota(\mathbf{v}, \hat{\mathbf{v}})(\bar{v}-\underline{v})\left(P_{k(\hat{\mathbf{v}}) \mid \underline{v}}-P_{k(\hat{\mathbf{v}}) \mid \bar{v}}\right) .
$$

Clearly, given that $\iota(\mathbf{v}, \hat{\mathbf{v}})>0, \bar{v}>\underline{v}$ and $k(\mathbf{v})=k(\hat{\mathbf{v}})$, we can simplify the last inequality in

$$
P_{k(\mathbf{v}) \mid \underline{v}} \leq P_{k(\mathbf{v}) \mid \bar{v}} .
$$

Hence, the set of flat IC constraints can be written compactly as in (8).

Actually, the downward, upward and flat IC constraints derived above do not exhaust the full set of IC constraints. In fact, while reporting a higher (or lower) valuation for some of the goods, the agent might also, simultaneously, permute the valuations of the remaining goods. Consider, as an example, $\mathbf{v}=(\underline{v}, \underline{v}, \bar{v}, \bar{v}, \bar{v})$ and $\hat{\mathbf{v}}=(\bar{v}, \bar{v}, \bar{v}, \underline{v}, \bar{v})$. In this case, we have $k(\hat{\mathbf{v}})=1<k(\mathbf{v})=2$, i.e. $\hat{\mathbf{v}}$ displays one $\bar{v}$ more than $\mathbf{v}$. Now, remove from $\mathbf{v}$ and $\hat{\mathbf{v}}$ the same entry so to obtain two vectors $\mathbf{w}$ and $\hat{\mathbf{w}}$ such that $k(\mathbf{w})=k(\hat{\mathbf{w}})$. In the example, we can remove either the first or the second entry and we obtain the vectors $\mathbf{w}=(\underline{v}, \bar{v}, \bar{v}, \bar{v})$ and $\hat{\mathbf{w}}=(\bar{v}, \bar{v}, \underline{v}, \bar{v})$. Notice that $\mathbf{w}$ and $\hat{\mathbf{w}}$ are not equal yet: $\hat{\mathbf{w}}$ is a permutation of $\mathbf{w}$; to pass from one to the other, one interchange must be performed (the first entry with the third). In symbols, we have $\iota(\mathbf{w}, \hat{\mathbf{w}})=1$ or, to avoid introducing further notation, $\iota(\mathbf{v}, \hat{\mathbf{v}})=1$.

In general, the interim utility of an agent who reports a higher valuation for some of the goods $(k(\hat{\mathbf{v}})<k(\mathbf{v}))$ and, possibly, permutes some of the other entries will be given by

$$
\begin{gathered}
U(\hat{\mathbf{v}} ; \mathbf{v})=\left(k(\mathbf{v})-k(\hat{\mathbf{v}}) \underline{v} P_{k(\hat{\mathbf{v}}) \mid \bar{v}}+(k(\hat{\mathbf{v}})-\iota(\mathbf{v}, \hat{\mathbf{v}})) \underline{v} P_{k(\hat{\mathbf{v}}) \mid \underline{v}}+(n-k(\mathbf{v})-\iota(\mathbf{v}, \hat{\mathbf{v}})) \bar{v} P_{k(\hat{\mathbf{v}}) \mid \bar{v}}\right. \\
+\iota(\mathbf{v}, \hat{\mathbf{v}}) \underline{v} P_{k(\hat{\mathbf{v}}) \mid \bar{v}}+\iota(\mathbf{v}, \hat{\mathbf{v}}) \bar{v} P_{k(\hat{\mathbf{v}}) \mid \underline{v}}+T_{k(\hat{\mathbf{v}})}
\end{gathered}
$$

which simplifies to

$$
U(\hat{\mathbf{v}} ; \mathbf{v})=U_{k(\hat{\mathbf{v}})}-(k(\mathbf{v})-k(\hat{\mathbf{v}}))(\bar{v}-\underline{v}) P_{k(\hat{\mathbf{v}}) \mid \bar{v}}+\iota(\mathbf{v}, \hat{\mathbf{v}})(\bar{v}-\underline{v})\left(P_{k(\hat{\mathbf{v}}) \mid \underline{v}}-P_{k(\hat{\mathbf{v}}) \mid \bar{v}}\right) .
$$

By incentive compatibility, we get

$$
U_{k(\mathbf{v})} \geq U_{k(\hat{\mathbf{v}})}-(k(\mathbf{v})-k(\hat{\mathbf{v}}))(\bar{v}-\underline{v}) P_{k(\hat{\mathbf{v}}) \mid \bar{v}}+\iota(\mathbf{v}, \hat{\mathbf{v}})(\bar{v}-\underline{v})\left(P_{k(\hat{\mathbf{v}}) \mid \underline{v}}-P_{k(\hat{\mathbf{v}}) \mid \bar{v}}\right),
$$

which is written compactly as

$$
\begin{gathered}
U_{k} \geq U_{k-i}-i(\bar{v}-\underline{v}) P_{k-i \mid \bar{v}}+\iota(\bar{v}-\underline{v})\left(P_{k-i \mid \underline{v}}-P_{k-i \mid \bar{v}}\right), \\
k=1, \ldots, n, \quad i=1, \ldots, k, \quad \iota=0, \ldots, \min \{k-i, n-k\} .
\end{gathered}
$$

When $\iota=0$, we obtain the upward IC constraints (7); when $i=0$, we obtain the flat IC constraints (8). Now, notice that, (7) and (8) together imply that the above inequalities are satisfied for all $\iota$.

By the same token, if an agent reports a lower valuation for some of the goods $(k(\hat{\mathbf{v}})>k(\mathbf{v}))$ and, possibly, permutes some of the other entries, incentive compatibility requires

$$
\begin{gathered}
U_{k} \geq U_{k+i}-i(\bar{v}-\underline{v}) P_{k-i \mid \bar{v}}+\iota(\bar{v}-\underline{v})\left(P_{k-i \mid \underline{v}}-P_{k-i \mid \bar{v}}\right), \\
k=1, \ldots, n, \quad i=1, \ldots, k, \quad \iota=0, \ldots, \min \{k-i, n-k\} .
\end{gathered}
$$

When $\iota=0$, we obtain the downward IC constraints (5); when $i=0$, we obtain the flat IC constraints (8). Again, (5) and (8) together imply that the above inequalities are satisfied for all $\iota$.

Therefore, the downward, upward and flat IC constraints defined in (5), (7) and (8) constitute the full set of relevant IC constraints. 


\section{B Proof of Proposition 2}

We start solving the reduced optimization problem

$$
\max _{(p, t)} 2 \sum_{k=0}^{n} f_{n, \alpha}(k) U_{k}
$$

subject to:

- $\operatorname{IR}_{n}$ (condition (9)),

- $\mathrm{IC}_{k}^{d}, k=0, \ldots, n-1$ (conditions (6)),

- BB (condition (10)),

- and the feasibility conditions $p(k|i ; j| l) \in[0,1]$, with $k, j=0, \ldots, n$ and $i, l \in$ $V^{13}$.

Then, in the last Step, we check whether also the ignored IC constraints are satisfied.

Notice that this problem does admit a solution: the objective function is continuous and the admissible set of decisions is clearly compact. What is not necessarily compact is the set of admissible transfers; however, notice that the problem involves only the reduced transfers $T_{k}$ and the set of admissible reduced transfers is indeed compact. Thus, by the Weierstrass maximum theorem, the problem admits a solution in terms of $T_{k}$ and therefore at least one in terms of non-reduced transfers $t(k ; j)$.

STEP 1: BB must be binding in the optimum.

Suppose not: then we could increase a little bit $T_{0}$ without violating any other constraint; by doing so, expected welfare would increase. The fact that BB is binding can be written as:

$$
2(1-\alpha)^{n} T_{0}=-\sum_{k=1}^{n} f_{n, \alpha}(k)\left[2 T_{k}+c\left(k P_{k \mid \underline{v}}+(n-k) P_{k \mid \bar{v}}\right)\right]-n(1-\alpha)^{n} c P_{0 \mid \bar{v}}
$$

and this pins down the optimal value of $T_{0}$.

Substituting back into the objective function and the other constraints, our reduced optimization problem becomes:

$$
\max _{(p, t)} \sum_{k=0}^{n} f_{n, \alpha}(k)\left[k(2 \underline{v}-c) P_{k \mid \underline{v}}+(n-k)(2 \bar{v}-c) P_{k \mid \bar{v}}\right]
$$

subject to $\operatorname{IR}_{n}$ (condition $\left.((9))\right), \mathrm{IC}_{k}^{d}, k=1, \ldots, n-1$ (conditions (6)), the feasibility conditions $p(k|i ; j| l) \in[0,1]$ and $\mathrm{IC}_{0}^{d}$, which now takes the following form:

$$
\begin{gathered}
n(1-\alpha)^{n}(2 \bar{v}-c) P_{0 \mid \bar{v}}-(1-\alpha)^{n-1}[2(1-\alpha) \bar{v}+n \alpha c]\left[(n-1) P_{1 \mid \bar{v}}+P_{1 \mid \underline{v}}\right] \\
-2(1-\alpha)^{n-1}[1+(n-1) \alpha] T_{1}-\sum_{k=2}^{n} f_{n, \alpha}(k)\left[\left(k P_{k \mid \underline{v}}+(n-k) P_{k \mid \bar{v}}\right) c+2 T_{k}\right] \geq 0
\end{gathered}
$$

Notice that transfers do not enter in the objective function anymore, but only in the constraints.

STEP 2: There is a solution in which $I R_{n}$ is binding.

Suppose there is a solution to the problem in which $\mathrm{IR}_{n}$ is not binding; take this solution and reduce $T_{n}$ so as to satisfy $\mathrm{IR}_{n}$ with equality. By doing so, $\mathrm{IC}_{0}^{d}$ and $\mathrm{IC}_{n-1}^{d}$ would be relaxed (and all the other IC constraints would be unaffected), while the

\footnotetext{
${ }^{13}$ Remember that, by definition, $p(0|\underline{v} ; j| l)=p(n|\bar{v} ; j| l)=p(k|i ; 0| \underline{v})=p(k|i ; n| \bar{v}) \equiv 0$.
} 
value of the objective function would be unchanged, i.e. we get another solution with $\mathrm{IR}_{n}$ binding. In such a solution, the value of $T_{n}$ is:

$$
T_{n}=-n \underline{v} P_{n \mid \underline{v}} .
$$

Substituting back, our problem reduces to:

$$
\max _{(p, t)} \sum_{k=0}^{n} f_{n, \alpha}(k)\left[k(2 \underline{v}-c) P_{k \mid \underline{v}}+(n-k)(2 \bar{v}-c) P_{k \mid \bar{v}}\right]
$$

subject to, $\mathrm{IC}_{k}^{d}, k=1, \ldots, n-2$ (conditions $\left.(6)\right)$, the feasibility conditions $p(k|i ; j| l) \in$ $[0,1], \mathrm{IC}_{0}^{d}$, which now takes the following form:

$$
\begin{gathered}
n(1-\alpha)^{n}(2 \bar{v}-c) P_{0 \mid \bar{v}}-(1-\alpha)^{n-1}[2(1-\alpha) \bar{v}+n \alpha c]\left[(n-1) P_{1 \mid \bar{v}}+P_{1 \mid \underline{v}}\right] \\
-2(1-\alpha)^{n-1}[1+(n-1) \alpha] T_{1}+n \alpha^{n}(2 \underline{v}-c) P_{n \mid \underline{v}}- \\
\quad \sum_{k=2}^{n-1} f_{n, \alpha}(k)\left[\left(k P_{k \mid \underline{v}}+(n-k) P_{k \mid \bar{v}}\right) c+2 T_{k}\right] \geq 0
\end{gathered}
$$

and $\mathrm{IC}_{n-1}^{d}$, which now takes the following form:

$$
(n-1) \underline{v} P_{n-1 \mid \underline{v}}-(\bar{v}-\underline{v}) P_{n \mid \underline{v}}+\bar{v} P_{n-1 \mid \bar{v}}+T_{n-1} \geq 0
$$

STEP 3: There is a solution in which $I C_{k}^{d}, k=1, \ldots, n-1$, are binding.

Suppose there exists a solution in which $\mathrm{IC}_{k}^{d}$ is not binding for some $k=1, \ldots, n-1$; then, we could reduce a little bit $T_{k}$ so as to satisfy $\mathrm{IC}_{k}^{d}$ with equality. By doing so, $\mathrm{IC}_{k-1}^{d}$ would still be satisfied as well as $\mathrm{IC}_{0}^{d}$, while the value of the objective function would be unchanged, i.e. we get a solution in which all $\mathrm{IC}_{k}^{d}, k=1, \ldots, n-1$ are binding.

Now, consider such a solution. The (binding) $\mathrm{IC}_{k}^{d}$ constraints, $k=1, \ldots, n-1$, can be written as

$$
\begin{gathered}
T_{k}=T_{k+1}-k \underline{v} P_{k \mid \underline{v}}-(n-k) \bar{v} P_{k \mid \bar{v}}+(\bar{v}+k \underline{v}) P_{k+1 \mid \underline{v}}+(n-k-1) \bar{v} P_{k+1 \mid \bar{v}}, k=1, \ldots, n-2, \\
T_{n-1}=-(n-1) \underline{v} P_{n-1 \mid \underline{v}}-\bar{v} P_{n-1 \mid \bar{v}}+(\bar{v}-\underline{v}) P_{n \mid \underline{v}} .
\end{gathered}
$$

Now, substitute recursively $T_{k}$ into $T_{k-1}$ starting from $k=n-1$. By doing so, we can determine the optimal values of the remaining transfers, which can be written as:

$$
T_{k}=(\bar{v}-\underline{v}) \sum_{j=k+1}^{n} P_{j \mid \underline{v}}-k \underline{v} P_{k \mid \underline{v}}-(n-k) \bar{v} P_{k \mid \bar{v}}, \quad k=1, \ldots, n-1 .
$$

Substituting back, our problem simplifies to:

$$
\max _{p} \sum_{k=0}^{n} f_{n, \alpha}(k)\left[k(2 \underline{v}-c) P_{k \mid \underline{v}}+(n-k)(2 \bar{v}-c) P_{k \mid \bar{v}}\right]
$$

subject to the feasibility conditions $p(k|i ; j| l) \in[0,1]$ and $\mathrm{IC}_{0}^{d}$, which, after some easy though tedious manipulations, can be written as:

$$
\sum_{k=0}^{n} f_{n, \alpha}(k)\left[k(2 \underline{v}-c) P_{k \mid \underline{v}}+(n-k)(2 \bar{v}-c) P_{k \mid \bar{v}}\right]-2(\bar{v}-\underline{v}) \sum_{k=1}^{n} F_{n, \alpha}(k-1) P_{k \mid \underline{v}} \geq 0 .
$$

STEP 4: Find the optimal values for $p(k|\underline{v} ; j| \underline{v})$ and $p(k|\bar{v} ; j| \bar{v})$.

Notice that, for all $k$, the coefficient attached to $P_{k \mid \underline{v}}$ is negative both in the objective function and in the $\mathrm{IC}_{0}^{d}$ constraint. Correspondingly, for all $k$, the coefficient attached 
to $P_{k \mid \bar{v}}$ is positive both in the objective function and in the $\mathrm{IC}_{0}^{d}$ constraint. One could then be mistakenly led to set $P_{k \mid \underline{v}}$ to the lowest possible level (that is 0 ) and $P_{k \mid \bar{v}}$ to the highest possible level (that is 1). But, as we remarked previously, $P_{k \mid \underline{v}}$ and $P_{k \mid \bar{v}}$ cannot be treated independently. When choosing the optimal decision, we must reason in terms of the non-reduced probabilities. Now, observe that, from the definition of reduced probabilities $(2), p(k|\underline{v} ; j| \underline{v})$ enters only in the definition of $P_{k \mid \underline{v}}$ and of $P_{j \mid \underline{v}}$. Correspondingly, $p(k|\bar{v} ; j| \bar{v})$ enters only in the definition of $P_{k \mid \bar{v}}$ and of $P_{j \mid \bar{v}}$. We can thus safely set

$$
p(k|\underline{v} ; j| \underline{v})=0, \quad k, j=1, \ldots, n
$$

and

$$
p(k|\bar{v} ; j| \bar{v})=1, \quad k, j=0, \ldots, n-1 .
$$

Instead, the mixed probabilities $p(k|\underline{v} ; j| \bar{v})$ enter both in $P_{k \mid v}$ and in $P_{j \mid \bar{v}}$ and thus their optimal values cannot be determined at this point. Using (17) and (18), the reduced probabilities simplify to:

$$
P_{k \mid \underline{v}}=\sum_{j=0}^{n} f_{n, \alpha}(j) \frac{n-j}{n} p(k|\underline{v} ; j| \bar{v}), \quad k=1, \ldots, n,
$$

and

$$
P_{k \mid \bar{v}}=\sum_{j=0}^{n} f_{n, \alpha}(j) \frac{j}{n} p(k|\bar{v} ; j| \underline{v})+(1-\alpha), \quad k=0, \ldots, n-1 .
$$

By the symmetry condition (1), the last equation can equivalently be written as

$$
P_{k \mid \bar{v}}=\sum_{j=0}^{n} f_{n, \alpha}(j) \frac{j}{n} p(j|\underline{v} ; k| \bar{v})+(1-\alpha), \quad k=0, \ldots, n-1 .
$$

Using (19) and (20), the objective function becomes:

$$
\begin{gathered}
(2 \underline{v}-c) \sum_{k=0}^{n} \sum_{j=0}^{n} f_{n, \alpha}(k) f_{n, \alpha}(j) \frac{k(n-j)}{n} p(k|\underline{v} ; j| \bar{v})+ \\
(2 \bar{v}-c) \sum_{k=0}^{n} \sum_{j=0}^{n} f_{n, \alpha}(k) f_{n, \alpha}(j) \frac{j(n-k)}{n} p(j|\underline{v} ; k| \bar{v})+n(1-\alpha)^{2}(2 \bar{v}-c) .
\end{gathered}
$$

Notice that the two double sums in the first two addends are identical. The objective function can thus be written as

$$
2(\bar{v}+\underline{v}-c) \sum_{k=0}^{n} \sum_{j=0}^{n} f_{n, \alpha}(k) f_{n, \alpha}(j) \frac{k(n-j)}{n} p(k|\underline{v} ; j| \bar{v})+n(1-\alpha)^{2}(2 \bar{v}-c),
$$

or simply

$$
2(\bar{v}+\underline{v}-c) \sum_{k=0}^{n} k f_{n, \alpha}(k) P_{k \mid \underline{v}}+n(1-\alpha)^{2}(2 \bar{v}-c) .
$$

Therefore, ignoring additive and positive multiplicative constants in the objective function, our problem can be written as:

$$
\max _{p} \sum_{k=1}^{n} k f_{n, \alpha}(k) P_{k \mid \underline{v}},
$$

subject to the feasibility conditions $p(k|i ; j| l) \in[0,1]$ and $\mathrm{IC}_{0}^{d}$, which now takes the following form:

$$
2(\bar{v}+\underline{v}-c) \sum_{k=1}^{n} k f_{n, \alpha}(k) P_{k \mid \underline{v}}-2(\bar{v}-\underline{v}) \sum_{k=1}^{n} F_{n, \alpha}(k-1) P_{k \mid \underline{v}}+n(1-\alpha)^{2}(2 \bar{v}-c) \geq 0
$$


(The expression for the $\mathrm{IC}_{0}^{d}$ constraint is obtained using (19) and (20) and after the same manipulations used to simplify the objective function.) Notice that only the reduced probabilities $P_{k \mid \underline{v}}$ are now involved.

SteP 5: Find the shape of the optimal reduced probabilities $P_{k \mid \underline{v}}$. The Langrangean for this linear programming problem is

$$
\begin{gathered}
L=\sum_{k=1}^{n} k f_{n, \alpha}(k) P_{k \mid \underline{v}}+ \\
\lambda\left(2(\bar{v}+\underline{v}-c) \sum_{k=1}^{n} k f_{n, \alpha}(k) P_{k \mid \underline{v}}-2(\bar{v}-\underline{v}) \sum_{k=1}^{n} F_{n, \alpha}(k-1) P_{k \mid \underline{v}}+n(1-\alpha)^{2}(2 \bar{v}-c)\right)+ \\
\sum_{k=1}^{n} \mu_{k}\left(1-\alpha-P_{k \mid \underline{v}}\right)+\sum_{k=1}^{n} \eta_{k} P_{k \mid \underline{v}}
\end{gathered}
$$

with Kuhn-Tucker (necessary and sufficient) conditions (for $k=1, \ldots, n$ ):

$$
k f_{n, \alpha}(k)+\lambda\left[2(\bar{v}+\underline{v}-c) k f_{n, \alpha}(k)-2(\bar{v}-\underline{v}) F_{n, \alpha}(k-1)\right]=\mu_{k}-\eta_{k}
$$

plus the complementary slackness conditions (for $k=1, \ldots, n$ ):

$$
\begin{gathered}
\lambda\left[2(\bar{v}+\underline{v}-c) \sum_{k=1}^{n} k f_{n, \alpha}(k) P_{k \mid \underline{v}}-2(\bar{v}-\underline{v}) \sum_{k=1}^{n} F_{n, \alpha}(k-1) P_{k \mid \underline{v}}\right. \\
\left.+n(1-\alpha)^{2}(2 \bar{v}-c)\right]=0 \\
\eta_{k} P_{k \mid \underline{v}}=0 \\
\mu_{k}\left(1-\alpha-P_{k \mid \underline{v}}\right)=0 .
\end{gathered}
$$

Notice first that, for all $k, \mu_{k}$ and $\eta_{k}$ cannot be both strictly greater than 0 (if they were, than either (23) or (24) would not be satisfied). This means that either $\mu_{k}>0$ and $\eta_{k}=0$ (in which case $P_{k \mid v}=1-\alpha$ ) or $\mu_{k}=0$ and $\eta_{k}>0$ (in which case $P_{k \mid \underline{v}}=0$ ) or $\mu_{k}=0$ and $\eta_{k}=0$ (in which case $P_{k \mid \underline{v}} \in[0,1-\alpha]$ ).

Second, notice that $\lambda>0$, i.e. $\mathrm{IC}_{0}^{d}$ is binding. To see this, suppose instead that $\lambda=0$. Then, for all $k$, we would have that the LHS of $(21)$ is strictly positive, meaning that $P_{k \mid \underline{v}}=1-\alpha$. But this violates $\mathrm{IC}_{0}^{d}$. In fact, by setting $P_{k \mid \underline{v}}=1-\alpha$ for all $k$ in $\mathrm{IC}_{0}^{d}$, we get

$$
\begin{gathered}
2(\bar{v}+\underline{v}-c) \sum_{k=1}^{n} k f_{n, \alpha}(k)-2(\bar{v}-\underline{v}) \sum_{k=1}^{n} F_{n, \alpha}(k-1)+n(1-\alpha)(2 \bar{v}-c) \geq 0 \\
2(\bar{v}+\underline{v}-c) n \alpha-2(\bar{v}-\underline{v}) n(1-\alpha)+n(1-\alpha)(2 \bar{v}-c) \geq 0 \\
2(\alpha \bar{v}+\underline{v})-(1+\alpha) c \geq 0
\end{gathered}
$$

which is satisfied if and only if $c \leq \underline{c}$, contradicting (12). Hence, there must be $k$ such that $P_{k \mid \underline{v}}<1-\alpha$. What we show in the following lemma is that, for all $i>0$, $P_{k+i \mid \underline{v}}=0$.

0 .

Lemma 4: Suppose that $\mu_{k}-\eta_{k} \leq 0$ for some $k=1, \ldots, n-1$. Then $\mu_{k+1}-\eta_{k+1}<$

Proof. If $\mu_{k}-\eta_{k} \leq 0$, then the expression between square brackets in the $k$-th Kuhn-Tucker condition (21) is necessarily strictly negative, i.e.

$$
\frac{\bar{v}+\underline{v}-c}{\bar{v}-\underline{v}}<\frac{F_{n, \alpha}(k-1)}{k f_{n, \alpha}(k)} .
$$


We claim that the expression between square brackets in the $(k+1)$-th Kuhn-Tucker condition is strictly negative as well, i.e. that

$$
\frac{\bar{v}+\underline{v}-c}{\bar{v}-\underline{v}}<\frac{F_{n, \alpha}(k)}{(k+1) f_{n, \alpha}(k+1)} .
$$

In order to do so, it is sufficient to show that

$$
\frac{F_{n, \alpha}(k-1)}{k f_{n, \alpha}(k)}<\frac{F_{n, \alpha}(k)}{(k+1) f_{n, \alpha}(k+1)} .
$$

Using the fact that $(k+1) f_{n, \alpha}(k+1)=(n-k) \frac{\alpha}{1-\alpha} f_{n, \alpha}(k)$ and that $F_{n, \alpha}(k-1)=$ $F_{n, \alpha}(k)-F_{n, \alpha}(k)$, this inequality can be equivalently written as

$$
\alpha(n-k) f_{n, \alpha}(k)-(n-k \alpha) F_{n, \alpha}(k)>0 .
$$

For $n \alpha \leq k$, this is obviously true. For $n \alpha>k$, we rewrite (25) as

$$
\frac{\alpha(n-k)}{n \alpha-k} f_{n, \alpha}(k)-F_{n, \alpha}(k)>0 .
$$

The LHS of (26) is strictly increasing in $k$. In fact

$$
\begin{gathered}
\frac{\alpha(n-k)}{n \alpha-k} f_{n, \alpha}(k)-F_{n, \alpha}(k)-\frac{\alpha(n-k+1)}{n \alpha-k+1} f_{n, \alpha}(k-1)-F_{n, \alpha}(k-1)= \\
\frac{1-\alpha}{n \alpha-k} k f_{n, \alpha}(k)-\frac{\alpha(n-k+1)}{n \alpha-k+1} f_{n, \alpha}(k-1)= \\
\frac{1}{n \alpha-k}-\frac{1}{n \alpha-k+1}>0 .
\end{gathered}
$$

Therefore, to check whether (26) is satisfied, it is sufficient to check whether $\frac{\alpha(n-1)}{n \alpha-1} f_{n, \alpha}(1)-$ $F_{n, \alpha}(1)>0$, which is always true.

We are now in the condition to prove the statement of the lemma. From the discussion above, the statement of the lemma is equivalent to say that

$$
\lambda \geq \frac{k f_{n, \alpha}(k)}{2(\bar{v}-\underline{v}) F_{n, \alpha}(k-1)-2(\bar{v}+\underline{v}-c) k f_{n, \alpha}(k)}
$$

implies

$$
\lambda>\frac{(k+1) f_{n, \alpha}(k+1)}{2(\bar{v}-\underline{v}) F_{n, \alpha}(k)-2(\bar{v}+\underline{v}-c)(k+1) f_{n, \alpha}(k+1)} .
$$

To prove this, it is sufficient to show that

$$
\frac{k f_{n, \alpha}(k)}{2(\bar{v}-\underline{v}) F_{n, \alpha}(k-1)-2(\bar{v}+\underline{v}-c) k f_{n, \alpha}(k)}>\frac{(k+1) f_{n, \alpha}(k+1)}{2(\bar{v}-\underline{v}) F_{n, \alpha}(k)-2(\bar{v}+\underline{v}-c)(k+1) f_{n, \alpha}(k+1)}
$$

which, after some manipulation, reduces to

$$
k F_{n, \alpha}(k)>(n-k) \frac{\alpha}{1-\alpha} F_{n, \alpha}(k-1) .
$$

This expression is equivalent to (25), which has already been shown to be true.

This implies that, if, for some $k, P_{k \mid \underline{v}}=0$, then also $P_{j \mid \underline{v}}=0$ for all $j>k$; by the same token, if, for some $k, P_{k \mid \underline{v}}=1-\alpha$, then also $P_{j \mid \underline{v}}=1-\alpha$ for all $j<k$.

STEP 6: Find the optimal cutoff value $\bar{k}$.

Now that we have determined the shape of the optimal reduced probabilities $P_{k \mid \underline{v}}$, what is left to do is to identify the optimal cutoff value $\bar{k}$. This is simply determined using the $\mathrm{IC}_{0}^{d}$ constraint, which has been shown to be binding. The idea is the following: we set $P_{j \mid \underline{v}}=1-\alpha$ for all $k \leq \bar{k}$ and $P_{j \mid \underline{v}}=0$ for all $k>\bar{k}$ and check whether the $\mathrm{IC}_{0}^{d}$ 
constraint is satisfied. For low values of $\bar{k}$ the constraint is satisfied, while for high values of $\bar{k}$ it is not. The optimal $\bar{k}$ is the one for which the $\operatorname{IC}_{0}^{d}$ constraint is still satisfied but it is not for $\bar{k}+1$.

By setting $P_{j \mid \underline{v}}=1-\alpha$ for all $k \leq \bar{k}$ and $P_{j \mid \underline{v}}=0$ for all $k>\bar{k}$, the $\mathrm{IC}_{0}^{d}$ constraint becomes

$$
2(\bar{v}+\underline{v}-c) \sum_{k=0}^{\bar{k}} k f_{n, \alpha}(k)-2(\bar{v}-\underline{v}) \sum_{k=0}^{\bar{k}} f_{n, \alpha}(k)(\bar{k}-k)+n(1-\alpha)(2 \bar{v}-c) \geq 0
$$

which can be written as (13). The optimal $\bar{k}$ is the highest number for which this inequality is still satisfied.

Step 7: Show that all the IC constraints are satisfied in the solution found.

Notice, first, that the optimal value of the reduced form probability $P_{k \mid \bar{v}}$ is independent of $k$ and is equal to:

$$
P_{k \mid \bar{v}}=\sum_{j=0}^{\bar{k}} f_{n, \alpha}(j) \frac{j}{n}+f_{n, \alpha}(\bar{k}+1) \frac{\bar{k}+1}{n} p_{\bar{k}+1|\bar{v} ; j| \underline{v}}+(1-\alpha), \quad k=0, \ldots, n-1 .
$$

Instead, the optimal value of the reduced form probability $P_{k \mid \underline{v}}$ is decreasing in $k$ and is at most $1-\alpha$. Therefore, for all $k, j, P_{k \mid \bar{v}}>P_{j \mid \underline{v}}$. This shows that the flat IC constraints (8) are satisfied.

To show that all the relevant downward IC constraints (5) hold, we use the fact that, in the solution, all the local downward IC constraints are binding, i.e.

$$
U_{k}=U_{k+1}+(\bar{v}-\underline{v}) P_{k+1 \mid \underline{v}}, \quad k=0, \ldots, n-1 .
$$

which implies

$$
U_{k}=U_{k+i}+(\bar{v}-\underline{v}) \sum_{j=1}^{i} P_{k+j \mid \underline{v}}, \quad k=0, \ldots, n-i, \quad i=1, \ldots, n-k .
$$

Because the optimal value of the reduced form probability $P_{k \mid \underline{v}}$ is decreasing in $k$, we have

$$
U_{k}=U_{k+i}+(\bar{v}-\underline{v}) \sum_{j=1}^{i} P_{k+j \mid \underline{v}} \geq U_{k+i}+i(\bar{v}-\underline{v}) P_{k+i \mid \underline{v}}
$$

which is (5).

Finally, using (28), (8) and (27) we have

$$
U_{k}=U_{k+i}+(\bar{v}-\underline{v}) \sum_{j=1}^{i} P_{k+j \mid \underline{v}} \leq U_{k+i}+(\bar{v}-\underline{v}) \sum_{j=1}^{i} p_{k+j \mid \bar{v}}=U_{k+i}+i(\bar{v}-\underline{v}) P_{k \mid \bar{v}}
$$

which implies that the upward IC constraints (7) are also satisfied. 


\section{References}

[1] W.J. Adams And J.L. Ellen. Commodity Bundling and the Burden of Monopoly. Quarterly Journal of Economics 90 (1976), 475-498.

[2] M. Armstrong. Multiproduct Nonlinear Pricing. Econometrica 64 (1996), $51-75$.

[3] M. Armstrong. Price Discrimination by a Many-Product Firm. The Review of Economic Studies 66 (1999), 151-168.

[4] M. Armstrong. Optimal Multi-Object Auctions. The Review of Economic Studies 67 (2000), 455-481.

[5] Y. Bakos and E. Brynjolfsson. Bundling Information Goods: Pricing, Profits and Efficiency. Management Science 45 (1999), 1613-1630.

[6] T. Börgers and P. Norman. A Note on Budget Balance under Interim Participation Constraints: The Case of Independent Types. Economic Theory 39 (2009), 477-489.

[7] H. Fang and P. Norman. Overcoming Participation Constraints. (Mimeo, 2006a).

[8] H. FAng And P. Norman. To bundle or not to bundle. RAND Journal of Economics 37 (2006b), 946-963.

[9] H. Fang and P. Norman. Optimal Provision of Multiple Excludable Public Goods. (Mimeo, 2008).

[10] X.J. Geng, M.B. Stinchcombe And A.B. Whinston. Bundling Information Goods of Decreasing Value. Management Science 51 (2005), 662-667.

[11] M.F. Hellwig. Optimal Income Taxation, Public-Goods Provision and Public Sector Pricing: A Contribution to the Foundations of Public Economics. Preprints of the Max Planck Institute for Research on Collective Goods (2004).

[12] M.F. Hellwig. The provision and pricing of excludable public goods: Ramsey-Boiteux pricing versus bundling. Journal of Public Economics 91 (2007), 511-540.

[13] M.O. JaCkson and H.F. Sonnenschein. Overcoming incentive constraints by linking decisions. Econometrica 75 (2007), 241-257.

[14] G.J. Mailath and A. Postlewaite. Asymmetric Information Bargaining Problems with Many Agents. The Review of Economic Studies 57 (1990), 351-367.

[15] R.P. McAfee, J. McMillan and M. Whinston. Multiproduct Monopoly, Commodity Bundling, and the Correlation of Values. Quarterly Journal of Economics 103 (1989), 371-383.

[16] R. Myerson. Optimal Auction Design. Mathematics of Operations Research 6 (1981), 58-73. 
[17] T.R. Palfrey. Bundling Decisions by a Multiproduct Monopolist with Incomplete Information. Econometrica 51 (1983), 463-484. 